\title{
MiR-30e and miR-181d control Radial Glia cell proliferation via HtrA1 modulation
}

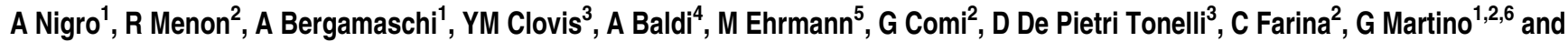 \\ L Muzio*,1,6
}

The precise mechanisms by which microRNAs (miRNAs) contribute to the dynamic regulation of gene expression during the forebrain development are still partly elusive. Here we show that the depletion of miRNAs in the cerebral cortex and hippocampus, via genetic inactivation of Dicer after the onset of forebrain neurogenesis, profoundly impairs the morphological and proliferative characteristics of neural stem and progenitor cells. The cytoarchitecture and self-renewal potential of radial glial (RG) cells located within the cerebral cortex and the hippocampus were profoundly altered, thus causing a significant derangement of both the post natal dorsal sub-ventricular zone and the dentate gyrus. This effect was attributed to the High-temperature requirement $A$ serine peptidase 1 (HtrA1) gene product whose overexpression in the developing forebrain recapitulated some of the aspects of the Dicer $^{-l-}$ phenotype. MiR-30e and miR-181d were identified as posttranscriptional negative regulators of $H$ trA1 by binding to its $3^{\prime}$ untranslated region. In vivo overexpression of miR-30e and miR-181d in Dicer ${ }^{-I-}$ forebrain rescued RG proliferation defects.

Cell Death and Disease (2012) 3, e360; doi:10.1038/cddis.2012.98; published online 2 August 2012

Subject Category: Neuroscience

During the early forebrain development neuroepithelial cells give rise to a distinct but related type of progenitor cells namely radial glial $(R G)$ cells, which rapidly proliferate to expand their number, and also generate neurons that delaminate from the germinal layers. As a consequence, the radial thickness of the cerebral cortex increases. ${ }^{1}$ The RG bipolar cell morphology - characterized by a narrow apical surface and a long basal process attached to the pial basement by end feet - contributes to RG functioning and guides neuronal cell migration. Thus, aberrant molecular processes affecting RG cell morphology might profoundly interfere with the proliferative potential of these cells, and in turn with proper development of the forebrain.

Although several evidence suggesting that transcription factors and diffusible signals influence RG self-renewal, morphology and differentiation, ${ }^{2,3}$ ensuing evidence indicates that also the posttranscriptional regulation of gene expression has a crucial role in the same events. MicroRNAs (miRNAs) have been found to exert essential regulatory functions in brain development. ${ }^{4}$ They are short (19-25-nt) noncoding RNAs, that regulate gene expression posttranscriptionally by nearly-perfect base-pairing with specific mRNA targets. ${ }^{5}$ Remarkably, miRNAs sequences, their expression patterns, and sequences of respective RNA targets are conserved during evolution, suggesting the ancient origin and the crucial function of miRNAs in conserved developmental processes. ${ }^{6}$ Although it has been recently estimated that the majority of mammalian mRNAs contains miRNAs binding sequences, the vast majority of these potential miRNAs targets still await experimental validation. ${ }^{7}$ In addition to their classical function of posttranscriptional regulators, miRNAs can also accelerate the decay of their target mRNAs by mediating mRNA destabilization. ${ }^{8}$

We characterized cell morphology and cell proliferation of RG cells in both cortical and hippocampal regions of Dicer (Dicer1) - i.e., type-III RNAse, essential for maturation of nearly all miRNAs ${ }^{9,10}$ - conditional knockout forebrains. By performing genome-wide transcriptome analysis of forebrains lacking miRNAs, we show that the High-temperature requirement $A$ serine peptidase 1 (HtrA1) is significantly increased in cortical and hippocampal RG cells upon miRNAs loss. By overexpressing HtrA1 in the wild-type forebrains, we recapitulated several aspects of the Dicer ${ }^{-1-}$ phenotype, including the derangement of $R G$ cell morphology and cell proliferation. Two miRNAs predicted by bioinformatic approaches were found to act on the $3^{\prime}$ UTR of HtrA1 and to modulate its

\footnotetext{
${ }^{1}$ Neuroimmunology Unit, INSpe, Division of Neuroscience, San Raffaele Scientific Institute, Milan, Italy; ${ }^{2}$ INSPE, Department of Neuroscience, San Raffaele Scientific Institute, Milan, Italy; ${ }^{3}$ Department of Neuroscience and Brain Technologies, Istituto Italiano di Tecnologia, Genova, Italy; ${ }^{4}$ Department of Biochemistry, section of pathology, Second University of Naples, Naples, Italy and ${ }^{5}$ Centre for Medical Biotechnology, Faculty of Biology University, Duisburg-Essen, Essen, Germany ${ }^{*}$ Corresponding author: L Muzio, Neuroimmunology Unit, INSpe, Division of Neuroscience, San Raffaele Scientific Institute, Via Olgettina no. 58, Dibit II-5 ${ }^{\circ}$ floor, Sector A4, 20132 Milan, Italy. Tel: +39 022643 4851; Fax: +39 022643 4855; E-mail: muzio.luca@ hsr.it

${ }^{6}$ Both authors joint senior authorship

Keywords: dicer; neurogenesis; HtrA1; miR-30e; miR-181d

Abbreviations: RG, radial glia; miRNA, microRNA; SVZ, subventricular zone; VZ, ventricular zone; DG, dentate gyrus; HtrA1, High-temperature requirement A serine peptidase 1; UTR, untranslated region; CR, Calretinin; SGZ, subgranular zone; BP, basal progenitor; IUE, in utero electroporation; BrdU, bromodeoxyuridineldU, iododeoxyuridine; CldU, Chlorodeoxyuridine; EdU, ethynyldeoxyuridine; Tgf- $\beta$, transforming growth factor- $\beta$; BMP, Bone Morphogenetic Protein; pH3, phospho-histone 3; WT, wild type; TUNEL, terminal dUTP nick-end labeling

Received 11.4.12; revised 25.6.12; accepted 25.6.12; Edited by A Verkhratsky
} 
expression in vivo during forebrain development. By reintroducing mimics encoding miR-30e and miR-181d in Dicer ${ }^{-1-}$ mice, we were able to revert Dicer $^{-1-}$ cell-proliferation defects.

\section{Results}

RG morphology and postnatal neurogenesis are altered in the VZ/SVZ of Gfap ${ }^{\text {Cre }}$ Dicer $^{\mathrm{f} / \mathrm{f}}$ mice. Gap ${ }^{\text {Cre }}$ mice $^{11}$ were crossed with Rosa26YFP reporter mice to assess the temporal and spatial expression of the Cre recombinase. ${ }^{12}$ At embryonic day $(E) 12.5$, the Cre-mediated recombination of the Rosa26YFP locus occurred in cells of the hippocampus (Supplementary Figure S1a), whereas at E13.5, the YFP was expressed by the vast majority of cells of the cortical wall, including cells located at the ventricular lining, possibly belonging to RG cell population (Supplementary Figure S1b). We next crossed mice carrying a floxed allele for Dicer $\left(\text { Dicer }^{f / f}\right)^{13}$ with Gfap ${ }^{\text {Cre }}$ mice to ablate miRNAs. The gross brain anatomy was maintained as shown by haematoxylin staining at E16.5, E18.5, postnatal day (P)15 and P40 (Supplementary Figures S2a-i). However, the hippocampus and the dentate gyrus (DG) were abnormally small in Gfap Cre Dicer $^{f / f}$ mice (Supplementary Figures S2b, d, f, h and i). Because early ablation of Dicer in the forebrain impairs the corticogenesis, ${ }^{14}$ we stained E14.5, E16.5, E18.5 brains for TuJ1 (Supplementary Figures S3a, c and e) and P15 and P40 brains for NeuN (Supplementary Figures S3g and i) to measure the thickness of the cortical wall. The neuronal layer of the cerebral cortex was reduced at P15 and P40 in mice lacking Dicer (Supplementary Figure S3). The hippocampal shrinkage was further confirmed by labeling E18.5 sections for Calretinin $(\mathrm{CR})^{15}$ and for $\alpha$-Crystallin ${ }^{16}$ (Supplementary Figures S4a-e). P15 Gfap $^{\text {Cre }}$ Dicer $^{\mathrm{f} / \mathrm{f}}$ brains showed a marked reduction of DG size (Supplementary Figure S2h) and the iper-cellularization of the dorsal sub-ventricular zone (SVZ) (Supplementary Figures S2c and g). The analysis of P40 brains confirmed these results (Supplementary Figure S2i).

Because neuronal cell survival is compromised in the absence of Dicer, ${ }^{14,17-19}$ we next evaluated cell death in Gfap $^{\text {Cre }}$ Dicer $^{f / f}$ forebrains at E16.5, E18.5 and P15 using Tunel staining. The number of apoptotic cells was similar at E16.5 in Gfap ${ }^{\text {Cre }}$ Dicer $^{f /+}$ (hereafter referred to as control mice) and Gfap ${ }^{\text {Cre }}$ Dicer $^{f / f}$ mice (control: $0.67 \pm 035$; Gfap ${ }^{\text {Cre }}$ Dicer $^{f / f}: 0.82 \pm 0.15$ Tunel $^{+} /$section, Supplementary Figures S2j and n). At E18.5 cell death was slightly, but significantly, increased in Gfap ${ }^{\text {Cre }}$ Dicer $^{f / f}$ mice (control: $0.94 \pm 0.22$; Gfap $^{\text {Cre }}$ Dicer $^{f / f}: 4.38 \pm 1.17$ Tunel $^{+} /$section, $P<0.01$, Supplementary Figures S2k and o). At P15 Gfap ${ }^{C r e}$ Dicer ${ }^{f / f}$ mice exhibited a modest increment of apoptotic cells (control: $0.65 \pm 0.29 ; \quad$ Gfap $^{\text {Cre }}$ Dicer $^{f / f}: 2.5 \pm 1.6$ Tunel $^{+} /$section, $P<0.05$, Supplementary Figures $\mathrm{S} 2 \mathrm{I}-\mathrm{q}$ ), that especially persisted in the dorsal SVZ (Supplementary Figures S2I and p), while undetectable within the subgranular zone (SGZ) of the Gfap ${ }^{C r e}$ Dicer $^{f / f}$ hippocampus (Supplementary Figures $\mathrm{S} 2 \mathrm{~m}$ and $\mathrm{q}$ ). Because a low frequency of cell death occurring in Gfap $^{\mathrm{Cre}}$ Dicer $^{f / f}$ mice may arise from incomplete Cremediated recombination of the Dicer ${ }^{f / f}$ locus, we confirmed recombination efficiency in Gfap $^{\text {Cre }}$ Dicer $^{f / f}$ Rosa26YFP brains. The vast majority of cortical and DG cells incorporating the S-phase tracer EdU were also $\mathrm{YFP}^{+}$(Supplementary Figures S5a and b). Approximately $94 \%$ of cortical cells and $96 \%$ of DG cells expressing the RG marker $R C 2^{20}$ were positive for the YFP (Supplementary Figures S5c-f).

We next studied RG cell proliferation in the Gfap Cre Dicer ${ }^{f / f}$ brains at E14.5, E16.5, E18.5, P15 and P40 by injecting $\mathrm{S}$-phase tracers. Cell proliferation was assayed in a series of consecutive bins (10 $\mu \mathrm{m}$ each) encompassing both E14.5 lateral and medial ventricular zone (VZ) and SVZ (Supplementary Figures S6a, b, e and f). At E14.5, cell cycle parameters - i.e., cell cycle length and growth fraction (GF) measured in control forebrains confirmed previously reported data (not shown). ${ }^{21}$ Gfap $^{\text {Cre }}$ Dicer $^{f / f}$ forebrains, however, did not show any alteration of S-phase tracers incorporation (Supplementary Figures S6c, d, g and h). We evaluated cell cycle parameters at E16.5 by pulsing mice with BrdU, EdU and IdU (Figures 1a and b). With the long labeling paradigm $(6 \mathrm{~h})$, we estimated a significant increment of the GF in Gfap ${ }^{\text {Cre }}$ Dicer $^{f / f}$ cortices (control: $48.6 \pm 1.9 \%$; Gfap ${ }^{\text {Cre }}$ Dicer $^{f / f}$ : $58.0 \pm 1.8 \%, P<0.001$, calculated in the first seven bins encompassing the VZ/SVZ) and a significant increment of labeled cells occupying outer bins of the VZ/SVZ (Figures 1c and d). Accordingly, the thickness of the cortical VZ/SVZ was increased in Gfap ${ }^{C r e}$ Dicer $^{f / f}$ mice, as shown by the distribution of $\mathrm{Ki}^{+} 7^{+}$cells (Figures 1e and f). Because cells of the outer VZ/SVZ might belong to basal progenitor (BP) cell population, we stained sections for the BP marker Tbr2, ${ }^{22}$ and EdU (Figures $1 \mathrm{~g}$ and $\mathrm{h}$ ). As previously reported, ${ }^{14}$ neither the total number of $\mathrm{Tbr}^{+}$cells nor percentages of Tbr2/EdU doublepositive cells were changed after miRNAs ablation (Figures $1 \mathrm{i}$ and j). At E18.5, the VZ/SVZ of control forebrains exhibited a significant reduction of the thickness, and the vast majority of proliferating cells were clustered in the first three bins of the VZ (Figure 1k). The GF of Gfap ${ }^{C r e}$ Dicer $^{f / f}$ mice, calculated for the $6 \mathrm{~h}$ labeling paradigm was significantly increased upon miRNAs deprivation (control: $57.1 \pm 2.4 \%$; Gfap ${ }^{C r e}$ Dicer $^{f / f}$ : $66.6 \pm 0.2 \%, P<0.05$, calculated in the first 3 bins). Moreover, a significant number of proliferating cells was misplaced in the second and the third bins of the VZ/SVZ, indicating that the cytoarchitecture is altered (Figures $11-n$ ). Because an aberrant accumulation of early-generated neurons might displace proliferating $R G$ cells from their proper location, causing the radial enlargement of the $\mathrm{VZ}$, we labeled sections for Ki67 and TuJ1. However, we did not observe any alteration of the TuJ1 ${ }^{+}$cell distribution within cortical VZ/SVZ of Gfap ${ }^{C r e}$ Dicer $^{f / f}$ mice (Supplementary Figures S7a-b), suggesting that VZ/SVZ enlargement was caused by proliferating RG cells. We studied RG cell morphology by in utero electroporating (IUE) plasmids encoding pCAG-Cherry vector in E16.5 control and Gfap $^{\text {Cre }}$ Dicer $^{f / f}$ mice. Cherry ${ }^{+}$cells in control VZs displayed morphological features of RG cells (Figure 10). In contrast, Cherry ${ }^{+}$cells, in Gfap ${ }^{C r e}$ Dicer $^{f / f}$ VZs, exhibited round-cell morphology, and many of them lost contacts with the apical membrane (Figure 1p). RG cell morphology was evaluated by labeling sections for Blbp and RC2. ${ }^{20,23} \mathrm{Blbp}^{+}$ cells of control mice exhibited cytoplasmatic bundles toward the pia and apical membranes, depicting a clear palisade structure (Supplementary Figure S7c). In Gfap ${ }^{\mathrm{Cre}}$ Dicer $^{\mathrm{f} / \mathrm{f}}$ forebrains, many Blbp ${ }^{+}$cells were placed in outer regions of 

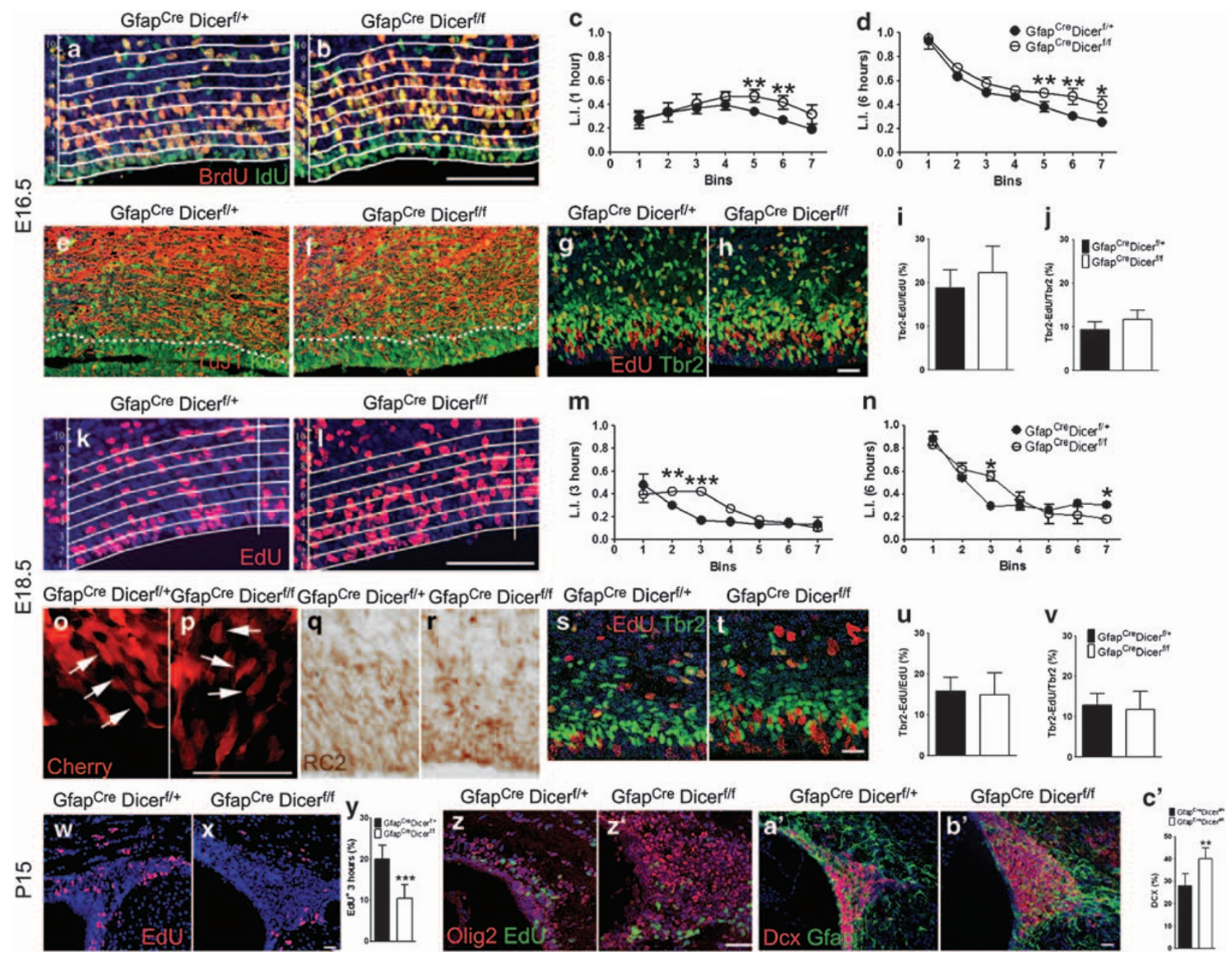

Figure 1 Gfap ${ }^{\mathrm{Cre}}$ mediated Dicer inactivation perturbed cell proliferation in the VZ/SVZ of the developing cerebral cortex. Cortical VZ/SVZ of E16.5 Gfap ${ }^{\mathrm{Cre}}$ Dicer/+ (a) and Gfap ${ }^{C r e} D_{i c e r} r^{f / f}(\mathbf{b})$ were labeled with BrdU (1 h) and IdU (6h), ( $n=3$ for each group). The analysis was conducted in a sector of $150 \mu \mathrm{m}$ wide bins that was subdivided into bins $10 \mu \mathrm{m}$ in height. They were numbered 1,2 and so on, from the ventricular lining outward (horizontal lines in panels (a) and (b)). One-hour BrdU administration preferentially labeled cells as the outer VZ/SVZ, while cumulative injections of IdU for 6-h-labeled cells that also reached the bin 1 . Fractions of positive cells in each bin are plotted in panels (c) and (d). Parallel sections were stained for TuJ1 and Ki67 (e, f), dotted lines indicate the boundary between the VZ/SVZ, and the mantle layer displaying $\mathrm{TuJ}^{+}$cells at high density. Double labeling for Tbr2 and EdU are shown in panels $(\mathbf{g})$ and $(\mathbf{h})$. Percentages ( \pm S.D.) of Tbr2/EdU ${ }^{+}$cells over the total number of EdU ${ }^{+}$cells are plotted in panel (i), whereas percentages ( \pm S.D.) of Tbr2/EdU ${ }^{+}$cells over the total number of Tbr2 ${ }^{+}$cells are shown in panel (j). E18.5 Gfap ${ }^{\text {Cre }}$ Dicer ${ }^{f /+}(\mathbf{k})$ and Gfap ${ }^{\text {Cre }}$ $\operatorname{Dicer}^{\mathrm{f} / f}$ (I) cortical sections were stained for EdU ( $\left.3 \mathrm{~h}\right),(n=3$ for each group). The VZ/SVZ was divided in consecutive $10 \mu \mathrm{m}$ thick bins and GFs calculated as above. The analysis of control brains revealed that the vast majority of EdU ${ }^{+}$cells were preferentially placed within the first two bins $(\mathbf{m}$ and $\mathbf{n})$, whereas Gfap ${ }^{\text {Cre }}$ Dicer/f brains exhibited more cells in outer bins ( $\mathbf{m}$ and $\mathbf{n})$. E18.5 Gfap ${ }^{\mathrm{Cre}} \operatorname{Dicer}^{\mathrm{f} /+}(\mathbf{o})$ and $\mathrm{Gfap}^{\mathrm{Cre}} \operatorname{Dicer}^{\mathrm{f} / \mathrm{f}}(\mathbf{p})$ received plasmids encoding the Cherry tracer at E16.5 $(n=3$ for each group). RG cells, of control mice, expressing the Cherry and displaying the elongated cell morphology of RG cells are shown in panel (o) (arrows). The thickness of the VZ/SVZ was increased in Gfap ${ }^{\mathrm{Cre}}$ Dice $^{\mathrm{f} / \mathrm{f}}$ and many Cherry ${ }^{+}$cells exhibited round-cell morphology and lost apical contacts with the basal membrane (arrows in (p)). Immunohistochemistry for RC2 on E18.5 control (q) and Gfap ${ }^{C r e} \operatorname{Dicer}^{f / f}(\mathbf{r})$ show altered palisade organization in the VZ of mice lacking mRNAs. Panels (s) and (t) show double staining for EdU and Tbr2. Percentages ( \pm S.D.) of double-labeled cells calculated as above are indicated in panels $(\mathbf{u})$ and $(\mathbf{v})$. P15 control $(\mathbf{w})$ and Gfap ${ }^{\mathrm{Cre}}$ Dicer $^{\mathrm{f} / \mathrm{f}}(\mathbf{x})$ brains $(n=3$ for each group) were pulsed with EdU for $6 \mathrm{~h}$ to label proliferating cells of the dorsal SVZ. Cell counts were done on one section every $300 \mu \mathrm{m}$ and data are indicated in panel (y). Adjacent sections were stained for Olig2 and EdU ( $z$ and $\left.z^{\prime}\right)$ and for GFAP and Dcx $\left(\mathbf{a}^{\prime}\right.$ and $\left.\mathbf{b}^{\prime}\right)$ to asses cell distribution into the SVZ. Dcx ${ }^{+}$cells are increased in the absence of miRNAs as shown in panel $\mathbf{c}^{\prime}$. ${ }^{\star} P<0.05 ;{ }^{* \star}, P<0.01 ;{ }^{\star \star *} P<0.001 ; t$ test. Scale bar $50 \mu \mathrm{m}$

VZ/SVZ, and often lost contacts with the apical membrane (Supplementary Figure S7d). Staining for RC2 confirmed these alterations in Gfap ${ }^{C r e}$ Dicer $^{f / f}$ forebrains (Figures $1 q$ and r). The total number of Tbr2 ${ }^{+}$cells and the percentage of Tbr2/ EdU double-positive cells were unaltered in Gfap ${ }^{C r e}$ Dicer $^{f / f}$ forebrains (Figures $1 \mathrm{~s}-\mathrm{v}$ ), indicating that proliferating cells of the outer bins did not belong to the BP cell population.

Because RG cells of the cerebral cortex are involved in the generation of adult proliferating neural stem cells that preferentially fate to the dorsal corner of the postnatal SVZ, ${ }^{24}$ we studied cell proliferation in these regions of P15 and P40 brains. The absolute number of proliferating cells and GF were significantly reduced in both P15 and P40 Gfap Cre Dicer $^{f / f}$ SVZs (Figures $1 w-y$ and Supplementary Figures S8a-c). By labeling P15 SVZs with Olig2, which labels parenchymal oligodendrocytes precursors and SVZ precursor cells, ${ }^{25}$ we found a significant accumulation of Olig2 in mice lacking Dicer (control: $23.7 \pm 2.8 \%$; Gfap ${ }^{\text {Cre }}$ Dicer $^{f / f}$ : $36.7 \pm 6 \%$, 
$P<0.05)$. However, only a limited fraction of these precursors were EdU ${ }^{+}$(control: $6.3 \pm 0.4 \%$; Gfap ${ }^{\text {Cre }}$ Dicer $^{f / f}: 3.6 \pm 2 \%$, $P<0.05$ ) (Figures $1 z$ and $z^{\prime}$ ). Accordingly, the number of $\mathrm{Dcx}^{+}$cells was significantly increased in the dorsal SVZ at P15 (Figures $1 a^{\prime}-c^{\prime}$ ). Thus, beside a substantial derangement of the RG cell morphology occurring during forebrain development, miRNA deprivation alters cell proliferation in post natal SVZs.

Cell proliferation is deranged in embryonic and postnatal DG of Gfap $^{\text {Cre }}$ Dicer $^{\mathrm{f} / \mathrm{f}}$ mice. We assayed cell proliferation in the hippocampus of Gfap ${ }^{\mathrm{Cre}}$ Dicer $^{f / f}$ by counting $\mathrm{IdU}^{+}$cells in a boxed area of $0.05 \mathrm{~mm}^{2}$ encompassing the SGZ and hilar zone of the DG. The total number of proliferating cells was significantly reduced at E16.5 (Figures 2a-c) and at E18.5 (Figures 2d-f). We then explored the expression of Tbr2 in the DG of Gfap Cre Dicer $^{f / f}$ mice. In the DG, we found that the number of Tbr2expressing cells was significantly reduced at E16.5 (Figures $2 \mathrm{j}-\mathrm{I}$ ) and at E18.5 (Figures $2 \mathrm{~m}-\mathrm{O}$ ). Depletion of proliferating cells was further confirmed by counting phospho-Histone 3 (pH3) positive cells at E18.5 (Figures $2 p-r)$. At P15 and P40 we found the most pronounced differences between control and Gfap $^{\mathrm{Cre}}$ Dicer $^{f / f}$ mice. Indeed, the number of EdU ${ }^{+}$cells were approximately eightfold reduced in the dorsal SVZ of Gfap $^{\text {Cre }}$ Dicer $^{f / f}$ mice at P15 and 3 fold reduced at P40 (Figures $2 g-i$ and Supplementary Figures S8d-f). We further observed a clear derangement of DG cytoarchitecture at P15, as shown by GFAP/Dcx labeling (Figures $2 \mathrm{~s}$ and $\mathrm{t}$ ).
These results show that the cytoarchitecture of DG in Gfap $^{C r e}$ Dicer $^{f / f}$ mice is severely deranged and cell proliferation in the SGZ is substantially reduced.

Gfap $^{\text {Cre }}$ Dicer $^{\mathrm{f} / \mathrm{f}}$ mice show forebrain transcriptome alterations. To identify the molecular mechanisms behind cellular and morphological alterations occurring in Gfap ${ }^{C r e}$ $D_{i c e r}{ }^{f / f}$ mice, we performed an unbiased transcriptome analysis of Gfap $^{\mathrm{Cre}} \mathrm{Dicer}^{f / f}$ cerebral cortex and hippocampus (Figure 3a). As we have detected significant changes of cellproliferation rates and the establishment of a clear alteration of the VZ/SVZ cytoarchitecture at the end of the gestation, tissues were collected at PO. Owing to the variable half-life (days to months) of miRNAs within the forebrain upon Dicer inactivation, ${ }^{26}$ we reasoned that miRNAs relevant for neurogenesis are not anymore expressed in proliferating niches of Gfap ${ }^{\text {Cre }}$ Dicer $^{f / f}$ forebrains at this time point. In both tissues, the majority of differentially expressed genes were upregulated in the Gfap ${ }^{\mathrm{Cre}}$ Dicer/f $^{f / f}$ mice compared with wild-type (WT) mice. In the cortex, 280 probes representing 258 genes were differentially expressed; among them, 161 probes (140 genes) were upregulated and 119 probes (118 genes) were downregulated. In the hippocampus, 510 probes (447 genes) were differentially expressed, among them 315 probes (264 genes) were upregulated and 195 probes (183 genes) were downregulated (Figure 3b). Ninetynine genes were coexpressed in both the hippocampus and the cortex. Surprisingly, 98 of them were similarly regulated (68 upregulated and 30 downregulated genes), thus
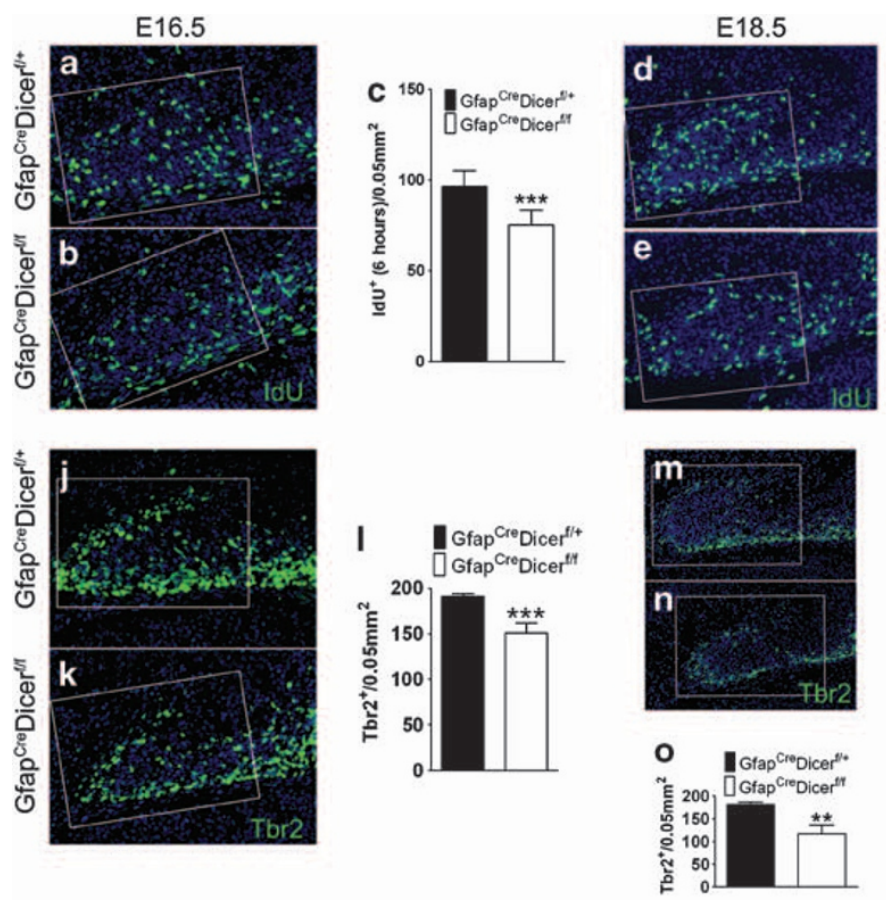
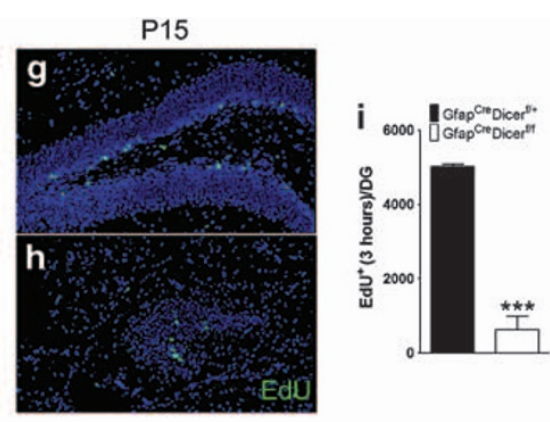
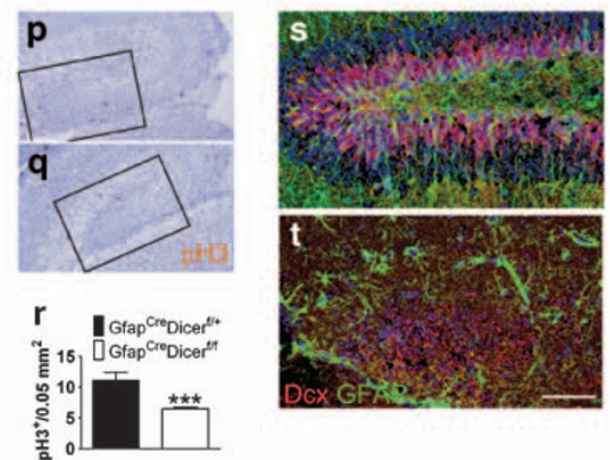

Figure 2 Gfap ${ }^{\mathrm{Cre}}$-mediated Dicer inactivation perturbed cell proliferation in the developing DG. Cell proliferation was evaluated in DGs derived from Gfap ${ }^{\mathrm{Cre}}$ Dicer $^{f /+}(\mathbf{a}, \mathbf{d}$ and $\mathbf{g}$ ) and Gfap ${ }^{C r e} \operatorname{Dicer}^{\mathrm{fff}}(\mathbf{b}, \mathbf{e}$ and $\mathbf{h})$ brains, that were pulsed with IdU or EdU tracers for 6 and $3 \mathrm{~h}$, respectively ( $n=3$ for each group). Boxed areas, encompassing DGs of E16.5 ( $\mathbf{a}$ and $\mathbf{~ b}), \mathrm{E} 18.5$ ( $\mathbf{d}$ and $\mathbf{e})$, indicate the regions $\left(0.05 \mathrm{~mm}^{2}\right)$ in which cells were counted, while cell proliferation was calculated in a region encompassing the entire DG of the P15 forebrain. Mean values ( \pm S.D.) of proliferating cells were counted on three independent samples per group and plotted in panels $\mathbf{c}$ and $\mathbf{f}$. Cell proliferation was substantially reduced at P15 ( $\mathbf{g}$ and $\mathbf{h}$ ) as shown in panel i. Adjacent sections from E16.5 ( $\mathbf{j}$ and $\mathbf{k}$ ) and from E18.5 $(\mathbf{m}$ and $\mathbf{n})$ brains were stained for Tbr2. Mean cell numbers $\left( \pm\right.$ S.D.) are plotted in panels $I$ and 0 . E18.5 brains were stained for $\mathrm{pH} 3(\mathbf{p}$ and $\mathbf{q})$ and $\mathrm{pH} 3^{+}$mean numbers $( \pm$S.D.) are plotted in panel $r$. P15 sections were further stained for GFAP and Dcx (s and t). ${ }^{* \star} P<0.01 ;{ }^{* \star \star} P<0.001 ; t$ test. Scale bar $100 \mu \mathrm{m}$ 
indicating that Dicer ablation in GFAP-expressing cells is characterized by conserved transcriptional changes in the cortex and in the hippocampus. To prioritize the genes within each signature, we applied the biomarker filter module of the Ingenuity software, focusing on genes related to the CNS and upregulated in the Gfap ${ }^{\mathrm{Cre}}$ Dicer $^{f / f}$ compared with WT mice with fold change $>2.5$. This filtering strategy highlighted eight genes commonly expressed in both regions. Among them, five genes were differentially expressed according to multiple probes in the microarray platform. We focused our attention on the upregulation of the HtrA1 gene, because its protein product binds a broad range of transforming growth factor (Tgf)- $\beta$ family proteins (e.g., BMP4, BMP2 and Tgf- $\beta 1),{ }^{27}$ which have critical roles in neurogenesis modulating either cell proliferation or cell survival. ${ }^{28-31}$

The overexpression of HtrA1 affects cell proliferation and cell morphology of the developing forebrain. RTand real-time PCR assays on PO Gfap ${ }^{\text {Cre }}$ Dicer $^{f / f}$ brains confirmed the upregulation of HtrA1 mRNA (Figures $4 \mathrm{a}-\mathrm{c}$ ). By radioactive in situ hybridization, we assayed HtrA1 expression. Although undetected at E10.5 (Figure 4d), HtrA1 expression was identified in E14.5 hippocampal and ganglionic VZ/SVZs (Figure 4e). WT cortical VZ/SVZ expressed HtrA1 at detectable levels at E18.5 (Figure 4f). Gfap Cre $D_{i c e r}{ }^{f / f}$ forebrains exhibited high HtrA1 levels in the cortical VZ/SVZ and, above all, in SGZ. In addition, ectopic HtrA1 expression in scattered cells of the cortical and hippocampal plates was noted (Figure 4g). HtrA1 operates as negative modulator of secreted BMPs by preventing protein binding to their cell receptors. ${ }^{27}$ The suppression of BMP activity, by knocking out BMP receptors, causes a severe reduction of DG. ${ }^{29,30}$ The overexpression of a constitutive active form of BmpR1a inhibits the expression of FoxG1 in the cortex, suggesting that BMPs act as negative regulators of FoxG1. ${ }^{31}$ E18.5 Gfap $^{\text {Cre }}$ Dicer $^{f / f}$ brains, exhibiting high levels of the BMPs-antagonist HtrA1 (Figure 4g), showed a significant upregulation of FoxG1 in the hippocampus (Figures $4 \mathrm{~h}-\mathrm{j}$ ). The level of phopsphorylated Smad transcription factors (pSmad1/5/8), which transduce BMP signalling, ${ }^{32}$ was reduced in Gfap $^{C r e}$ Dicer $^{f / f}$ mice (Figures $4 \mathrm{k}$ and I). Accordingly, Msx1, which is positively regulated by the BMP signaling, ${ }^{33,34}$ was downregulated in Gfap Cre Dicer ${ }^{f / t}$ DGs (Figures $4 \mathrm{~m}$ and $\mathrm{n}$ ). Because BmpR1a and BmpR1b double knockout mice exhibit decreased expression of the Prospero homeobox gene Prox1 in DG granule cells, ${ }^{29}$ we probed P15 Gfap ${ }^{\text {Cre }}$ Dicer $^{f / f}$ forebrains for Prox1. The total number of Prox $1^{+}$cells was decreased in Gfap ${ }^{C r e}$ Dicer $^{f / f}$ forebrains (Figures 40 and $p$ ). We next performed IUE of HtrA1-expressing plasmids in the cortex. ${ }^{35}$ E13.5 cortical cells received $\mathrm{pCAG}-\mathrm{GFP}$ with or without plasmids encoding the mouse HtrA1, and embryos were subsequently collected at E15.5. Efficient overexpression of HtrA1 was confirmed by probing targeted forebrains with a specific antibody for HtrA1 (not shown). ${ }^{36}$ VZ-restricted cells receiving GFP plasmids exhibited the classical palisade organization. By contrast, the VZ cytoarchitecture of mice receiving HtrA1 was severely disturbed. Indeed, a large number of targeted cells acquired round-cell morphology and lost their proper connections with the apical membrane (Figures $5 a$ and $b$ ). Double staining for GFP and RC2 (Figures 5c-h) or for GFP and Blbp (Figures $5 \mathrm{i}$ and j) substantially confirmed $R G$ cell cytoarchitectural alterations in brains receiving HtrA1. Based on EdU incorporation at the day of sacrifice, we noticed that HtrA1 overexpression impaired cell proliferation in the VZ (Figures $5 \mathrm{k}-\mathrm{m})$, slightly, but significantly, reduced the expression of Tbr2 (Figures $5 n-p$ ), but did not affect cell survival (Supplementary Figures S9a-d). We performed IUE of HtrA1 in the dorsal forebrain at E14.5. Embryos were then collected at E17.5 and cell proliferation was outlined by EdU labeling. Cell proliferation was significantly reduced in hippocampal cells receiving HtrA1 plasmids (Figures $5 q-s$ ), and also the expression of Tbr2 was diminished (Figures $5 t-v$ ).

Mir-30e and miR-181d interact with the $3^{\prime}$ UTR of the HtrA1 mRNA. To identify miRNAs regulating HtrA1 expression in the forebrain, we performed IUE of $3^{\prime} U T R$ of the mouse HtrA1 cloned into a Renilla luciferase vector (Rluc-HtrA1-3'UTR) in E13.5 cerebral cortices (Figure 6a). In order to measure the normalized Rluc-HtrA1-3'UTR activity in cortical proliferating cells, forebrains were collected $8 \mathrm{~h}$ after IUE. Luciferase activity was significantly diminished in cortices receiving the Rluc-HtrA1-3'UTR plasmid, suggesting the existence of regulatory miRNAs acting on the HtrA13'UTR (Figure 6a). A bioinformatic strategy based on mirSVR scoring in miRBase database was used to predict candidate miRNAs, targeting the HtrA1 transcript. Upon scanning of 522 nucleotides of the HtrA1-3'UTR region, 16 putative miRNAs were identified, among which miR-149 had the highest score (Figure 6b). Furthermore, we performed miRNA enrichment analysis on the transcriptional targets upregulated in the cortex or hippocampus of Gfap ${ }^{\mathrm{Cre}}$ Dicer $^{f / f}$ mice using Genecodis, and isolated 118 and 353 significantly enriched miRNAs for cortex and hippocampus data sets, respectively. MiR-30e and miR-181d appeared in the predictions based on both the HtrA1-3'UTR region and the Gfap $^{\text {Cre }}$ Dicer $^{f / f}$ transcriptomes. In addition, 11 miRNAs, including the high score miR-149, were common to the mirSVR prediction and the hippocampus data sets. We confirmed that miR-30e, -149 and $-181 d$ were reduced by almost $70 \%$ in Gfap $^{\text {Cre }}$ Dicer $^{f / f}$ cortical and hippocampal micro-dissections (Figures 6c-e). To prove miRNA binding to $3^{\prime}$ UTR of HtrA1, we cotransfected HeK-293T cells with Rluc-HtrA1-3'UTR plasmids and mimics encoding miR-30e, -149 and $-181 d$. Controls received mimics encoding the unrelated miRNA cel-miR-67 and transfection efficiency were estimated by using unrelated mimics conjugated with a fluorescent dye (Figure 6f). Mimics encoding miR-30e and $181 \mathrm{~d}$ induced a significant reduction of luciferase expression already at low doses, whereas mimics encoding miR-149 reduced the expression of the luciferase mainly at high dosage (Figure $6 \mathrm{~g}$ ). We confirmed that predicted miR-30e and $-181 d$ target sites in HtrA1-3' UTR are critical for repression of HtrA1 expression by introducing point mutations that disrupt the base-pairing of both predicted target sites into Rluc-HtrA1-3'UTR construct (Figure 6g).

IUE of miR-30e and miR-181d rescued cell proliferation defects in Gfap ${ }^{\text {Cre }}$ Dicer $^{\mathrm{f} / \mathrm{f}}$ mice. We next asked, whether 


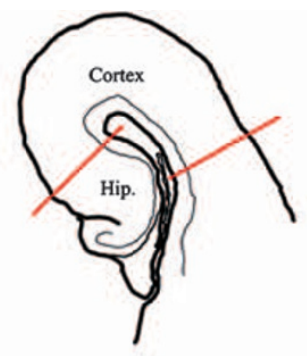

b

Illumina MouseWG-6 v2 : 45281 probes

O Background substraction

- Cubic Spline normalization

O Detection p-value based filtering$$
\downarrow
$$

Detected probes : 17992

WT vs Gfap ${ }^{\text {cro Dicer }}$

DIFFERENTIAL GENE EXPRESSION ANALYSIS

CORTEX
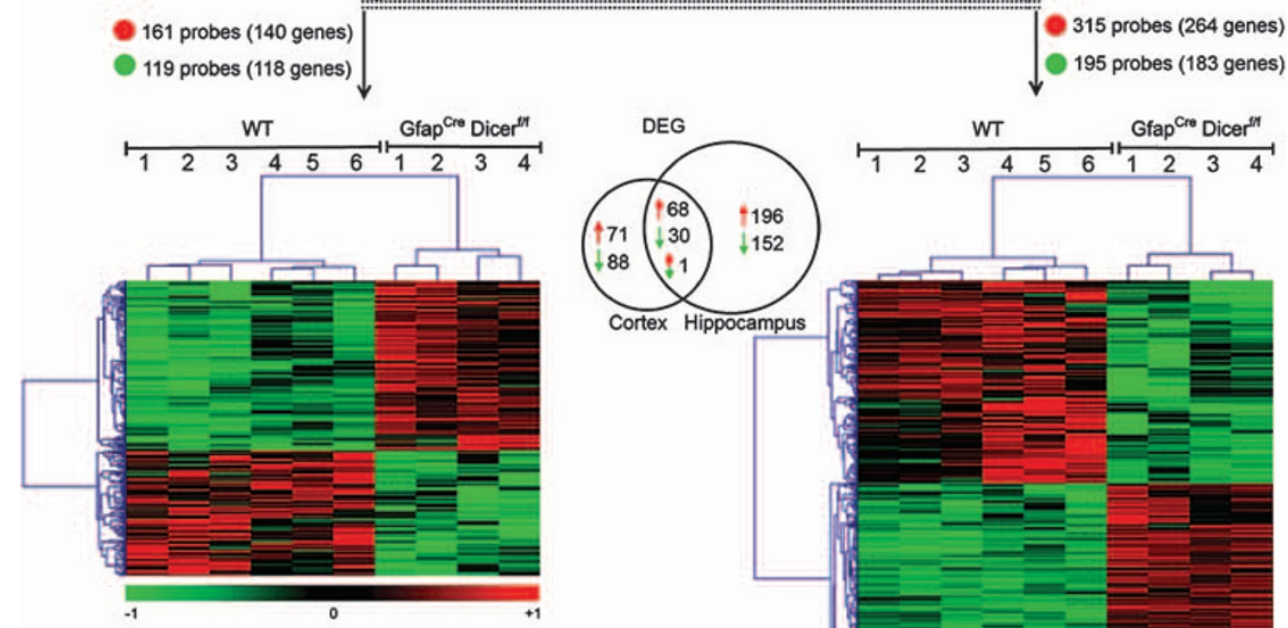

Cortex Hippocampus
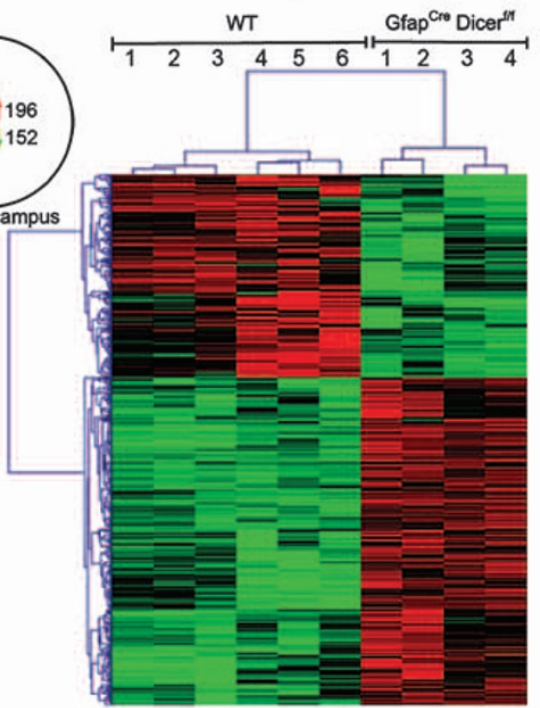

INGENUITY BIOMARKER FILTER ANALYSIS
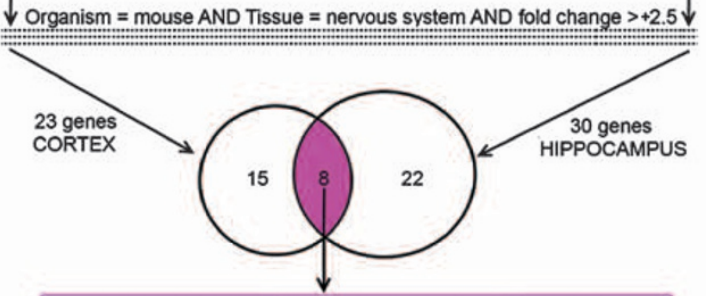

Bves*/ Col22a1 / Fbln2\% HtrA1\%/ Itgb1*/ K if1b / Nfatc3*/ Tac2

*Represented by multiple probes

Figure 3 Transcriptomics highlighted shared signatures in the cortex and hippocampus of Gfap Cre Dicer/f transgenic animals. Panel a shows a schematic coronal section from P0 forebrain indicating sampled micro-dissected regions included in the transcriptome analysis. Panel $\mathbf{b}$ shows the workflow of transcriptome analysis. The raw data from 45281 probes were subjected to background subtraction, normalization and detection filtering. The differential expression analysis between the wild-type (WT) versus Gfap Cre Dicer ${ }^{\mathrm{f} / f}$ was conducted by LIMMA method in Bioconductor package with $P$-value $<0.01$ and fold change threshold of \pm 1.7 . The number of differently regulated probes along with the genes are given for both the cortex and the hippocampus. The direction of differential expression is represented by red (upregulation) and green (downregulation) color in the Gfap ${ }^{\mathrm{Cre}}$ Dicer $^{\mathrm{f} / \mathrm{f}}$ compared with WT. The unsupervised hierarchical clustering of the samples classified properly the Gfap ${ }^{\mathrm{Cre}}$ Dicer $^{\mathrm{f} / \mathrm{f}}$ from WT specimens. The upper Venn diagram shows the shared and unique differentially expressed genes (DEG) in cortex and hippocampus with the direction of regulation. Finally, upregulated probes were subjected to biomarker filter analysis in Ingenuity software with the parameters of organism $=$ mouse, tissue $=$ nervous systems and fold change $>+2.5$, separately for the cortex and the hippocampus. This resulted in 23 and 30 genes, respectively in the cortex and the hippocampus. The Venn diagram in bottom highlights (in pink color) the eight common biomarkers in the cortex and the hippocampus, which are listed on the bottom. Asterisk highlights those genes significantly regulated by multiple probes in the array

the reintroduction of miR-30e and $-181 \mathrm{~d}$ in Gfap ${ }^{\mathrm{Cre}}$ Dicer $^{\mathrm{f} / \mathrm{f}}$ forebrains can revert cell proliferation defects that we encountered in medial cortical field and in the DG. We performed IUE of mimics encoding miR-30e and $-181 \mathrm{~d}$ along with pCAG-Cherry plasmids in the medial cortical field of both control and Gfap Cre Dicer/f/f forebrains at E14.5 (Figures 7a-d). Because cel-miR-67 did not regulate the expression of HtrA1 in vitro, we included in our analysis WT brains electroporated with this miRNA (Figure 7e). Brains were pulsed with EdU at E17.5 and assayed for cell 

Cortex Hippocampus
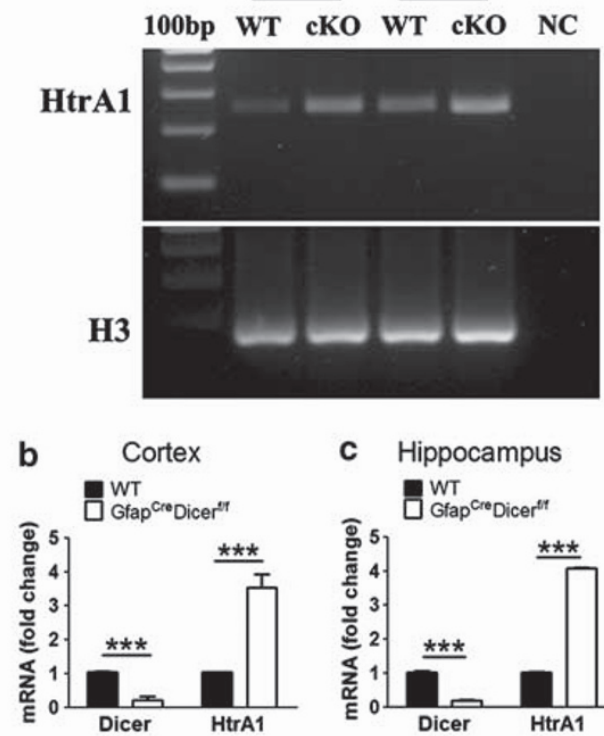

C Hippocampus

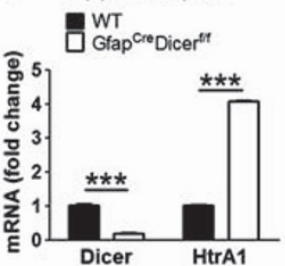

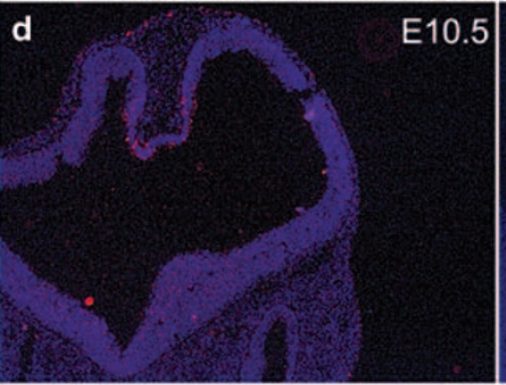
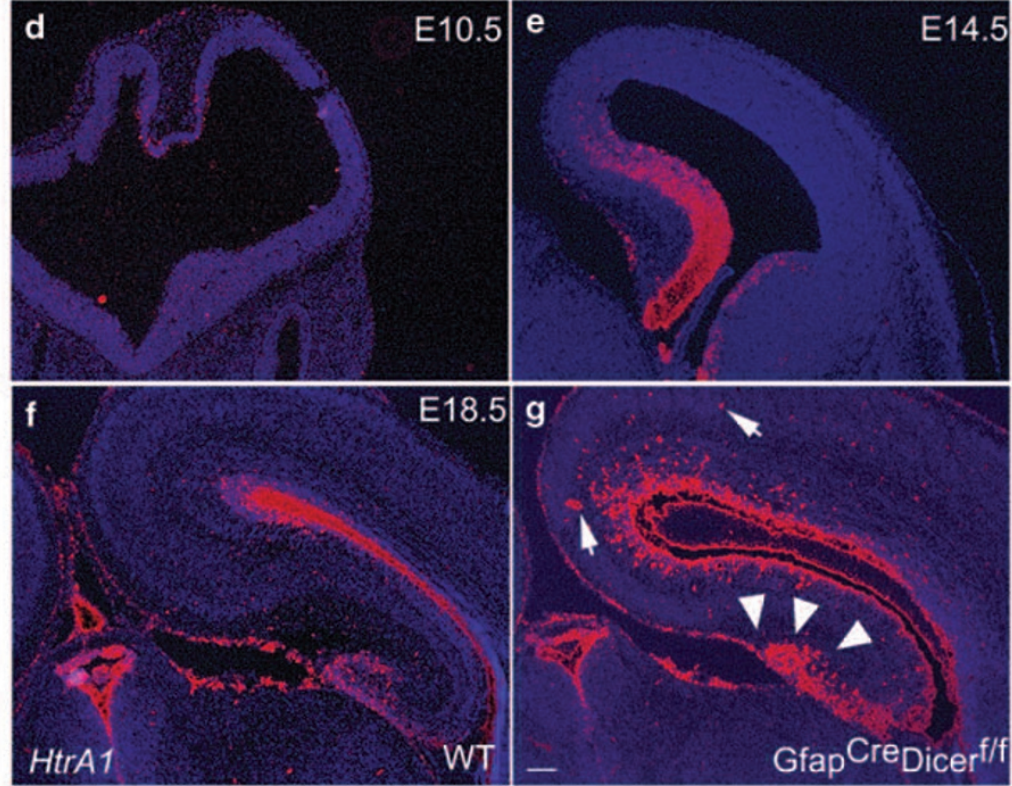

WT

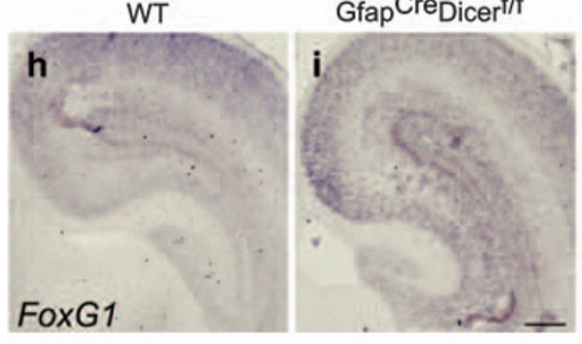

WT

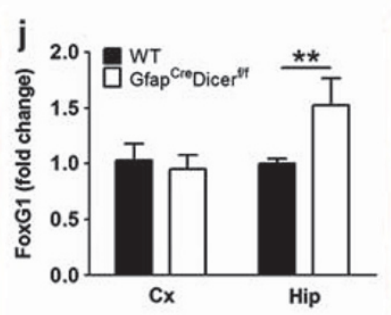

GfapCreDicer ${ }^{\mathrm{f} / \mathrm{f}}$
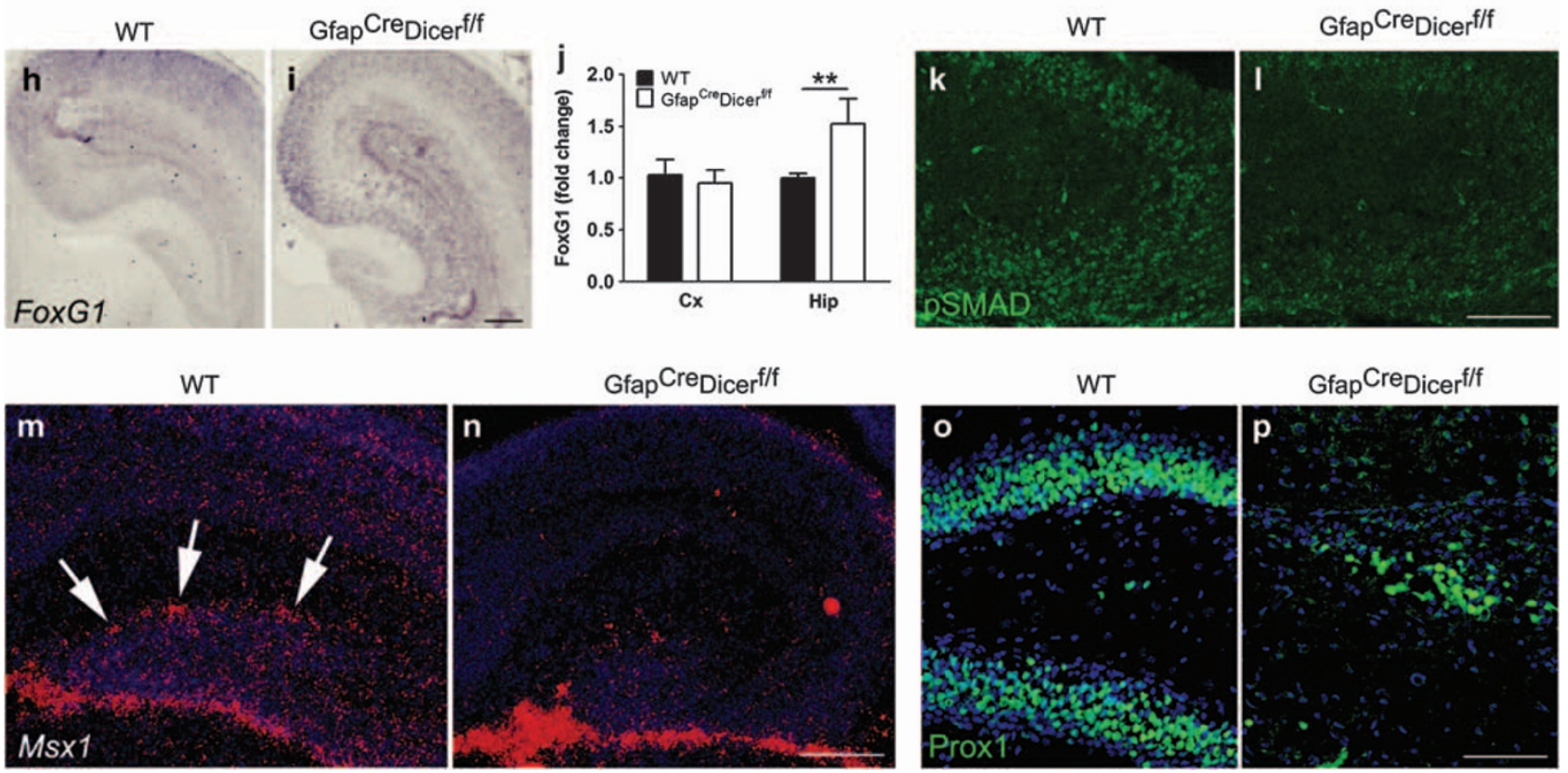

Figure $4 \mathrm{HtrA} 1$ increased expression in Gfap ${ }^{\mathrm{Cre}}$ Dicer $^{\mathrm{t} / \mathrm{f}}$ forebrains. Total RNA extracts from WT and Gfap ${ }^{\text {Cre }}$ Dicer ${ }^{\mathrm{ff}}$ forebrains $(n=3$ for each group) were used for RT-PCR-mediated detection of HtrA1. Panel a shows a representative electrophoresis for HtrA1 (252 bp) and H3 (189 bp) amplicons. Real-time PCR detection of Dicer and $H t r A 1 \mathrm{mRNAs}$ are shown in panels $\mathbf{b}$ and $\mathbf{c}(n=3$ for each group). In situ detection of $H t r A 1 \mathrm{mRNA}$ was done by radioactive in situ hybridization (d-g). HtrA1 was undetectable within WT forebrains at E10.5 (d, $n=3)$, whereas robust expression levels were detected within the VZ/SVZ of the WT E14.5 hippocampus and in ganglionic eminences $(\mathbf{e}, n=3)$. At E18.5 HtrA1 expression was mainly found within the VZ/SVZ of the cerebral cortex, hippocampus and in the DG (f). E18.5 Gfap ${ }^{\text {Cre }}$ Dicer ${ }^{f / /}$ mice $(n=4)$ exhibited high expression levels of HtrA1 in the VZ/SVZ of the cerebral cortex, hippocampus (g) and DG (arrowheads in g). Interestingly, HtrA1 expression was found within ectopic cells of both the developing cortex and the hippocampus (arrows in $\mathbf{g}$ ). FoxG1 expression, measured by in situ hybridization ( $n=4$ for each group, h, i) and by real-time PCR ( $n=3$ for each group, j) was increased in the hippocampus of E18.5 Gfap ${ }^{\mathrm{Cre}}$ Dicer $^{\mathrm{f} / \mathrm{t}}$ mice. Coronal sections through hippocampus of E16.5 in a WT (k) and a Gfap ${ }^{\mathrm{Cre}}$ Dicer ${ }^{f / t}$ mutant (I), immunostained for pSmad1/5/8, transcription factors activated downstream of BMP signaling. The expression of $p S m a d 1 / 5 / 8$ was reduced in mice lacking Dicer ( $n=3$ for each group) The expression of Msx1, revealed by radioactive in situ hybridization, was reduced in the DG of Gfap ${ }^{\text {Cre }}$ Dicer ${ }^{f / f}$ mice $(n=3$ for each group, $\mathbf{m}, \mathbf{n})$. Prox1 expression levels were greatly reduced in $\mathrm{P} 15 \operatorname{Gfap}^{\mathrm{Cre}} \operatorname{Dicer}^{\mathrm{f} / \mathrm{f}}(\mathbf{0}, \mathbf{p}) .{ }^{* \star} P<0.01 ;{ }^{* * \star} P<0.001 ; t$ test. Scale bar $100 \mu \mathrm{m}$

proliferation. The overexpression of mimics encoding either miR-30e or $-181 d$ in control mice resulted in proliferation levels similar to WT forebrains receiving cel-miR-67 (Figure $7 f$ ), suggesting that the saturation of miR-30e and -
$181 \mathrm{~d}$ was already reached. By contrast, the reintroduction of miR-30e and -181d in Gfap ${ }^{C r e}$ Dicer $^{f / f}$ cells significantly increased the fraction of EdU ${ }^{+}$cells scored in medial cortical germinal niches (Figure 7f). These results suggest that both 

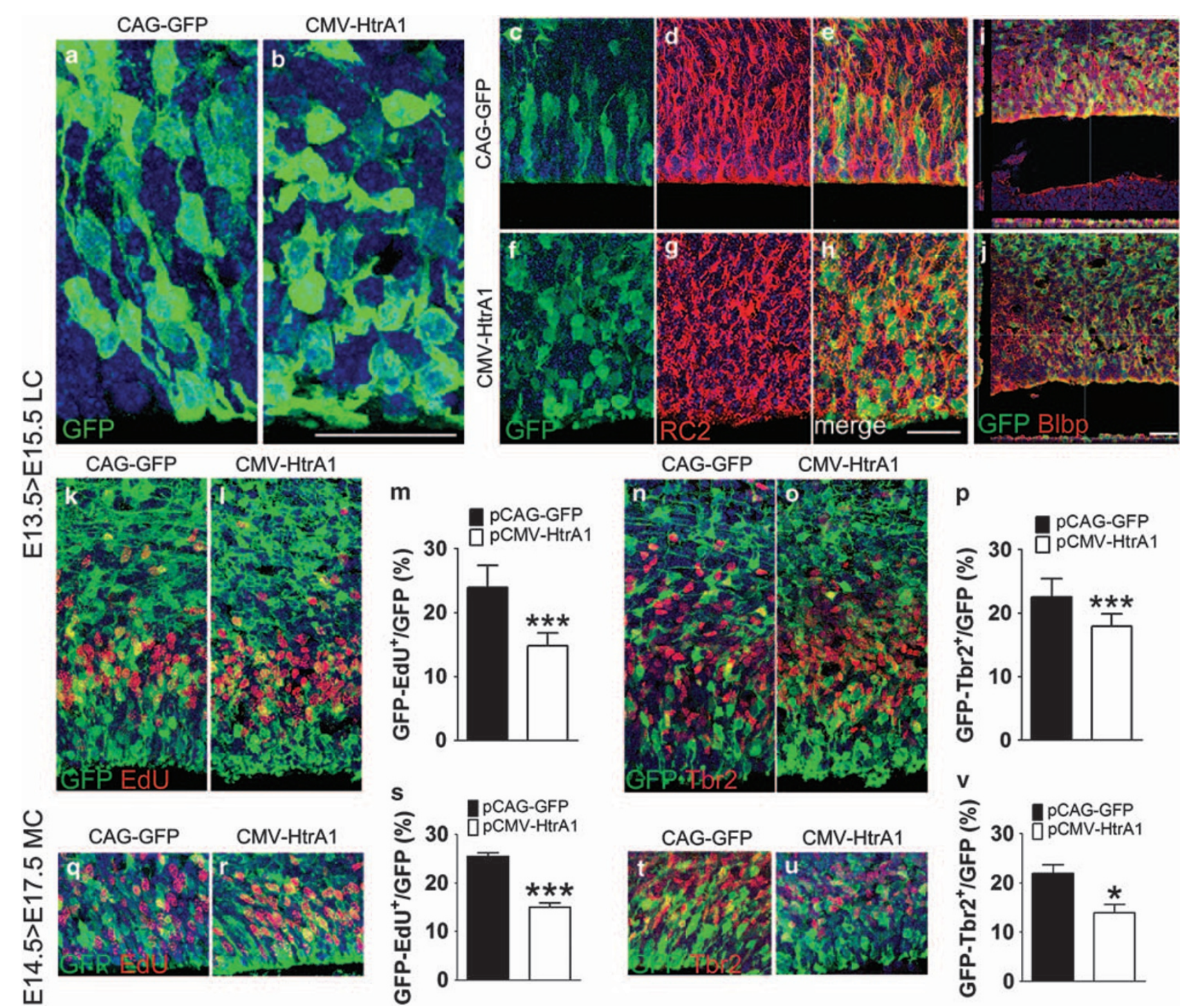

Figure 5 HtrA1 overexpression in WT brains altered RG cell morphology and impaired cell proliferation. pCAG-GFP plasmids $(\mathbf{a}, \mathbf{c}, \mathbf{d}, \mathbf{e}, \mathbf{i}, \mathbf{k}$ and $\mathbf{n})$, with or without plasmids encoding $H \operatorname{trA} \mathbf{1}$ (b, $\mathbf{f}, \mathbf{g}, \mathbf{h}, \mathbf{j}, \mathbf{I}$ and $\mathbf{~}$ ) were electroporated in the cerebral cortex at E13.5 ( $n=6$ for each group). Brains were left to develop in utero and then collected after $48 \mathrm{~h}$. Cell proliferation was outlined by pulsing mice with EdU for $1 \mathrm{~h}$ at the time of the euthanizing. The overexpression of HtrA1 altered RG cell morphology (a and $\mathrm{b}$ ). Double staining for GFP and RC2 confirmed alterations of the classical palisade morphology in forebrains receiving HtrA1 plasmids (f-h) when compared with controls receiving only pCAG-GFP plasmids (c-e). Panels $\mathbf{i}$ and $\mathbf{j}$ show confocal stacks of sections labeled for GFP and Blbp. Sections double labeled for EdU and GFP are shown in panels $\mathbf{k}$ and $\mathbf{I}$. Percentages ( \pm S.D.) of double-positive cells are indicated in panel $\mathbf{m}$. Parallel sections were stained for GFP and Tbr2 ( $n$ and 0$)$ and percentages ( \pm S.D.) of GFP/Tbr2 double-positive cells are indicated in panel p. E14.5 WT embryos were electroporated with pCAG-GFP plasmids (q and $\mathbf{t}$ ), with or without plasmids encoding HtrA1 ( $r$ and $u$ ) in medial cortical fields ( $n=5$ for each group). Samples were collected at E17.5, pulsed with EdU for $1 \mathrm{~h}$ and only forebrains displaying GFP ${ }^{+}$cells in the medial cortex were included in the analysis. Sections were stained for GFP and EdU ( $\mathbf{q}$ and $\mathbf{r}$ ) and percentages ( \pm S.D.) of GFP/EdU double-positive cells are indicated in panel $\mathbf{s}$. Parallel sections were stained for GFP and Tbr2 (t and $u$ ) and percentages ( \pm S.D.) of Tbr2/GFP double-positive cells are indicated in panel $\mathbf{v} .{ }^{*} P<0.05 ;{ }^{* * \star} P<0.001$; $t$ test. Scale bar $50 \mu \mathrm{m}$

miR-30e and -181d can efficiently inhibit HtrA1 expression and, above all, can increase cell proliferation rates in cells of the medial cortical field of the brain.

\section{Discussion}

Several experimental evidence indicates that miRNAs exert a critical function during early forebrain development. ${ }^{14,18,19}$ MiRNAs ablation, before the onset of the neurogenesis, is irrefutably accompanied by severe derangement of neurogenesis and increasing wave of cell death. ${ }^{14,17,18}$ Mice lacking Dicer ${ }^{13}$ exhibit several functional and molecular alterations of neurogenesis that include the following: the mis-expression of specific RG cell markers (Sox9, ErbB2 and Nestin), ${ }^{17}$ migratory defects, and the derangement of the cortical plate ${ }^{14}$ and the hippocampus. ${ }^{37}$ Although this complex phenotype might derive from cell proliferation defects, the contribution of miRNAs to RG cell proliferation/ self renewal is still missing. In this study, we explored RG cell proliferation in the cerebral cortex and hippocampus of Gfap $^{\mathrm{Cre}}$ Dicer $^{f / f}$ mice. Cell proliferation was severely impaired in the DG of Dicer null brains, confirming that miRNAs are 


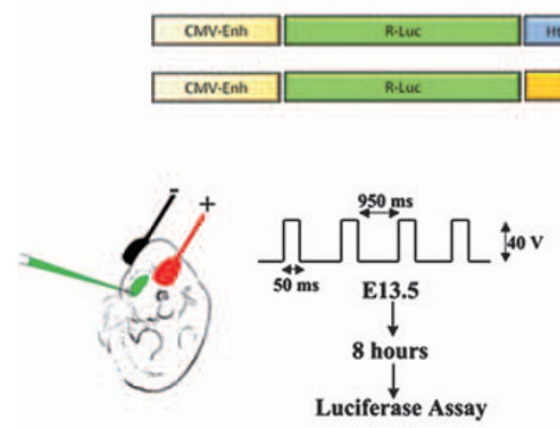

C

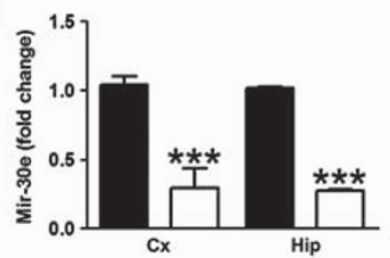

f

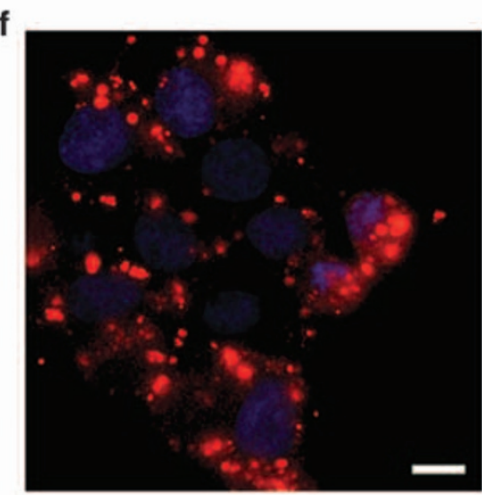

b
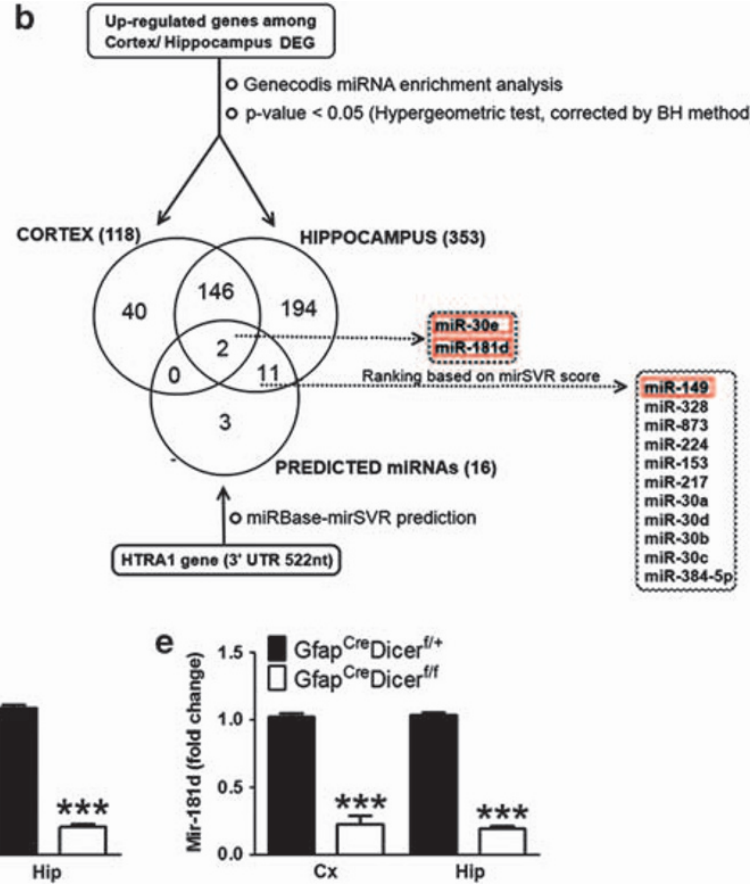

g
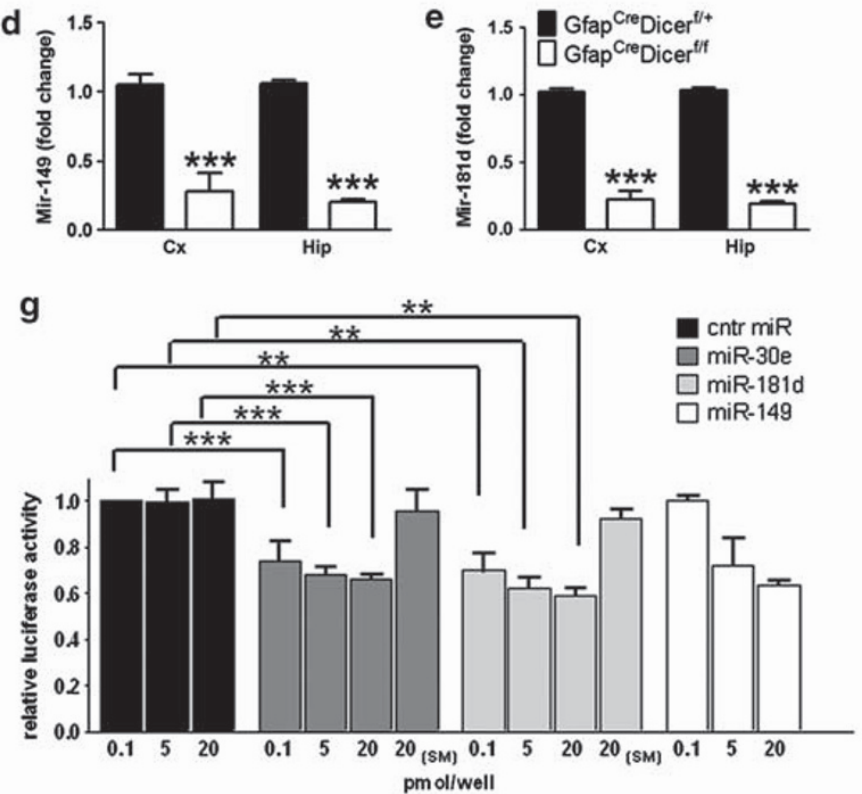

Figure 6 miR-30e and miR-181d interact with the $3^{\prime} U T R$ of HtrA1. The entire $3^{\prime}$ UTR of HtrA1 was cloned at the $3^{\prime}$ of Renilla luciferase (RLuc) expression plasmid (pCAGRluc-HtrA1-3'UTR vector) and electroporated in E13.5 forebrains along with pCAG-Fluc (firefly luciferase) and pCAG-mCherry. Control brains received empty RLuc plasmids (pCAG-RLuc-empty) ( $n=10$ mice per group) and pCAG-Fluc and pCAG-mCherry. Embryos were collected after $8 \mathrm{~h}$ and used for luciferase detection (a). Normalized percentages ( \pm S.D.) of luciferase are indicated in histogram of panel $\mathbf{a}$. The panel $\mathbf{b}$ shows the workflow of miRNA enrichment analysis and prediction. The upregulated genes in cortex (140 genes) and hippocampus (264 genes) were used for miRNA enrichment analysis, using Genecodis tool, with the $P$-value threshold of $<0.05$. There were 188 and 353 enriched miRNAs, respectively, in the cortex and the hippocampus. In the bottom, miRNA prediction for HtrA1 gene, using mirSVR score (miRBase database), is given. There were 16 miRNAs predicted as candidates capable of binding at the $3^{\prime}$ UTR region (522 nucleotides) of HtrA1 gene. The Venn diagram shows the overlap of enriched and predicted miRNAs, which are listed on the right side in dashed boxes. Two miRNAs, namely, miR-30e and -181d, were common to all the three comparisons. Further, ranking of 11 miRNAs common to the mirSVR prediction and the hippocampus is listed. Notably, mir-149 had the highest rank in the mirSVR prediction. Highlighted with red borders are the experimentally validated miRNAs. Hippocampal and cortical microdissections from Gfap ${ }^{\mathrm{Cre}}$ Dicer $^{f /+}$ and Gfap ${ }^{\mathrm{Cre}}$ Dicer ${ }^{f / t}$ mice $(n=4$ forebrains for each group) were used to measure the relative expression of miR-30e (c), miR-149 (d) and miR-181d (e). MiRNA double-strand mimics encoding miR-30e, -149 and -181d were transfected $(0.1,5$ and 20 pmol/well) in 293T cells along with pCAG-Rluc-HtrA1-3'UTR and pcDNA3.1-luc-firefly plasmids. We used, as control, mimics encoding the unrelated miRNA cel-miR-67 (it is referred to as cntr-miR). We included pCAG-Rluc-HtrA1-3'UTR plasmids displaying point mutations for the target sites of miR-30e and -181d in the $3^{\prime}$ UTR of HtrA1. Luciferase activities (mean values calculated over four independent experiments \pm S.D.) were measured after $48 \mathrm{~h}$ and plotted in panel $\mathbf{g}$. Efficiency of transfection was estimated by transfecting 293T cells with Dy547-labeled miRIDIAN microRNA Mimic Transfection Control (f). ${ }^{* *} P<0.01 ;{ }^{* * *} P<0.001 ; t$ test. Scale bar $10 \mu \mathrm{m}$

necessary for the hippocampal development. ${ }^{37}$ However, the examination of cortical RG cells also evidenced the presence of cytoarchitectural and cell proliferation defects. Cell proliferation is severely reduced at $\mathrm{P} 15$ and $\mathrm{P} 40$, indicating that miRNAs are essential for the maintenance of long-term proliferating $R G$ cells fated to these germinal niches. Apoptosis is the most widely observed phenotype occurring after miRNAs inactivation. ${ }^{14,17,37}$ However, apoptosis is slightly increased in Gfap $^{\mathrm{Cre}} \mathrm{Dicer}^{f / f}$ mice, suggesting that cell death occurring in the absence of miRNAs might take part in the establishment of Gfap $^{\text {Cre }}$ Dicer $^{f / f}$ phenotype, but its contribution is only marginal. MiRNAs inactivation induced a significant disorganization of cortical RG cells and many of them were placed at the outer VZ/SVZ, however, this did not 

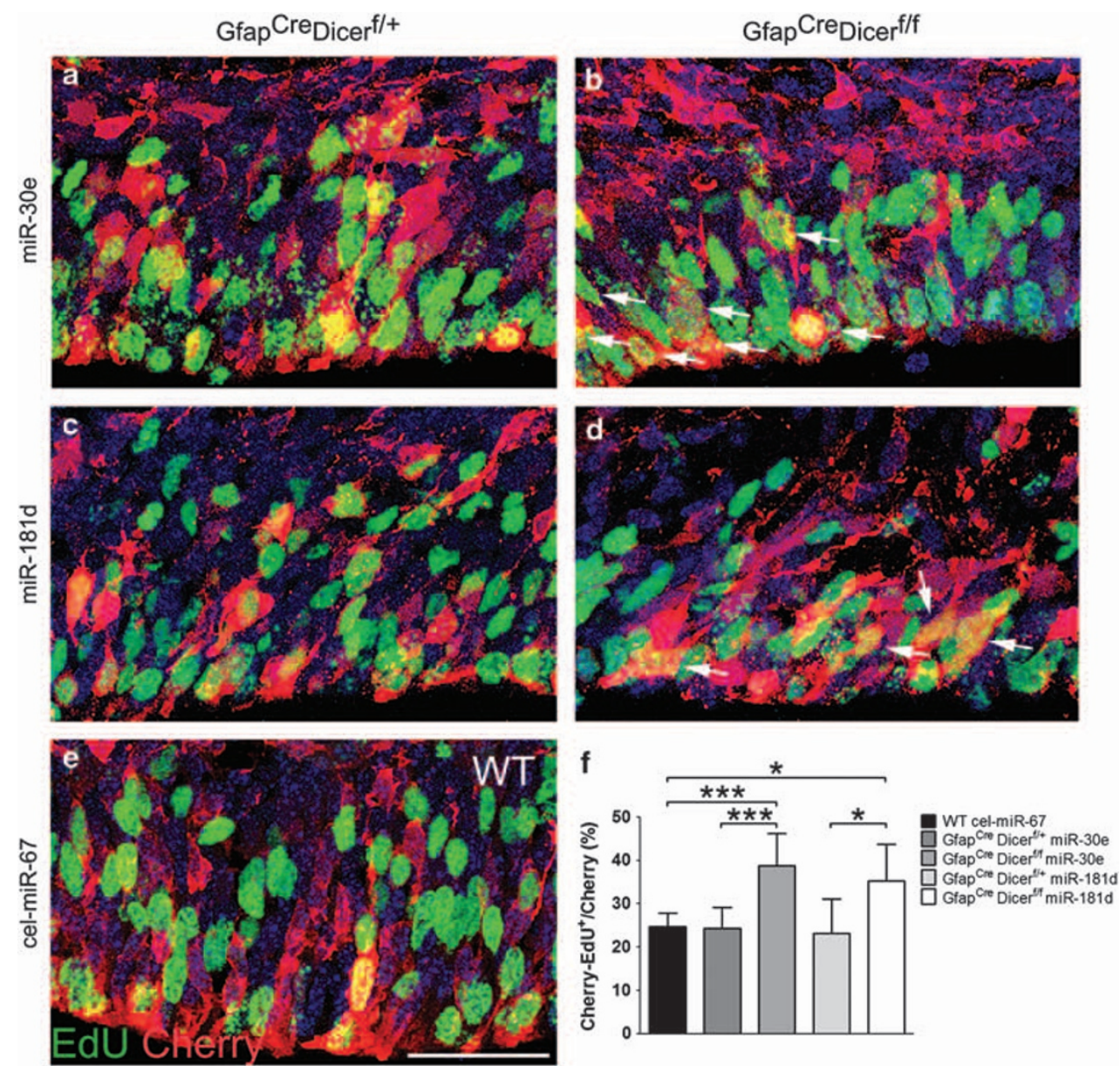

Figure 7 MiR-30e and miR-181d overexpression restored cell proliferation in Gfap ${ }^{\text {Cre }}$ Dicer $^{\text {r/f }}$ medial cortical fields. E14.5 Gfap ${ }^{C r e}$ Dice $^{f /+}(\mathbf{a}, \mathbf{c})$ and Gfap ${ }^{\mathrm{Cre}}$ Dice $^{\mathrm{fff}}(\mathbf{b}, \mathbf{d})$ mice received mimics encoding miR-30e $(\mathbf{a}, \mathbf{b})$ or $-181 \mathbf{d}(\mathbf{c}, \mathbf{d})$, along with pCAG-Cherry plasmids in the medial cortical field by IUE ( $n=3$ per group). Forebrains were left to develop in utero until E17.5, pulsed with EdU for $6 \mathrm{~h}$ and then euthanized. Each experiments were accompanied by WT forebrains receiving the unrelated miRNA cel-miR-67 and pCAG-Cherry plasmids $(n=4, \mathbf{e})$. Cell counts were done in the medial cortical field and percentages ( \pm S.E.M.) of EdU/Cherry double-positive cells (arrows in $\mathbf{b}$, d) over the total number of Cherry ${ }^{+}$cells are shown in panel f. ${ }^{*} P<0.05 ;{ }^{* *} P<0.001$; Anova and $t$ test. Scale bar $50 \mu \mathrm{m}$

reflect increasing numbers of cells expressing Tbr2 or TuJ1, suggesting that miRNAs deprivation might not affect cell differentiation.

By whole transcriptome analysis, we compared set of genes increased in cortical and hippocampal regions and we identified the HtrA1 gene product that belongs to a family of genes that were firstly identified in bacteria. ${ }^{38}$ In mammals, alterations of HtrA genes have been associated with cancerogenesis and the overexpression of a transgene codifying for HTRA1 inhibits tumor progression. ${ }^{39} \mathrm{HtrA1}$ can interfere with secreted $\operatorname{Tgf} \beta$ and BMPs proteins, ${ }^{27}$ and operates as microtubule-associated protein, modulating cell motility and cell polarity. ${ }^{40}$ Therefore, the high levels of HtrA1, which we measured in Gfap ${ }^{C r e}$ Dicer $^{f / f}$ forebrains, might affect BMP signalling causing a relevant disruption of proper forebrain development. Accordingly, increasing levels of HtrA1 were accompanied with a significant reduction of BMP targets: Prox1, pSmad $1 / 5 / 8^{29}$ and Msx $1 .{ }^{33}$ IUE of HtrA1 in WT cerebral cortices recapitulated morphological and functional defects observed in Gfap ${ }^{\text {Cre }}$ Dicer/f $^{f / f} \mathrm{RG}$ cells. We next identified putative target sequences in the $3^{\prime} U T R$ of HtrA1 for miR-30e, -149 and $-181 \mathrm{~d}$, and we further demonstrated that miR-30e and $-181 \mathrm{~d}$ can interact with the HtrA1-3'UTR. Because miRNAs can also modulate mRNAs destabilization $^{8}$ and our ongoing work shows that HtrA1 mRNAs is increased in mice lacking Dicer, it is reasonable to think that both miRNAs can regulate HtrA1 mRNAs stability. We finally tested their functional role by overexpressing mimics encoding miR-30e and miR-181d in RG cells of the Gfap $^{C r e}$ Dicer $^{f / f}$ forebrain, demonstrating that they can rescue cell proliferation defects that we observed in this genotype (Supplementary Figure S10).

\section{Materials and Methods}

Transgenic mouse lines. Mice were maintained in pathogen-free conditions at San Raffaele Hospital mouse facility (Milan, Italy) and at Italian institute of technology (IIT), Genova, Italy. All efforts were made to minimize animal suffering and to reduce the number of mice used in accordance with the European Communities Council Directive of November 24, 1986 (86/609/EEC). All animal experimental protocols were approved by the Ethics Review Committee for Animal Experimentation of the Italian Ministry of Health. Procedures were performed according to the guidelines of the Institutional Animal Care and Use Committee of 
the San Raffaele Scientific Institute (protocol number 329/2007) and of IIT Ethical Committee (protocol number 7050/11). Dicer ${ }^{f / f}$ mouse line was provided by Dr. Harfe, $^{13}$ Gfap $^{\text {Cre }}$ transgenic mouse line ${ }^{11}$ and Rosa26YFP transgenic reporter mice $^{12}$ were provided by Jackson laboratories. Transgenic mice were repeatedly backcrossed onto C57BL/6 (Charles River) mice and were genotyped by PCR as previously described. ${ }^{11-13}$ For specific control experiments we used wild type (WT) age-matched C57BL/6 mice. Noon of the vaginal plug date was day 0.5 in timed pregnancies. Pregnant females were killed by cervical dislocation at appropriate time points and brains were collected in ice-cold PBS as previously described. ${ }^{41}$ Brains were then fixed with $4 \%$ paraformaldehyde in PBS pH 7.2 for $12 \mathrm{~h}$ at $+4{ }^{\circ} \mathrm{C}$ and cryoprotected for $24 \mathrm{~h}$ in $30 \%$ Sucrose (Sigma, St. Louis, MO, USA) in PBS at $+4{ }^{\circ} \mathrm{C}$. Before the sacrifice, pregnant dams received multiple injections of S-phase tracers - i.e., BrdU, IdU, CldU, (Sigma) and EdU (Invitrogen, Carlsbad, CA, USA) at the concentration of $100 \mathrm{mg} / \mathrm{kg}$. Post natal mice were killed by anesthetic overdose and transcardially perfused with $4 \%$ paraformaldehyde in PBS pH 7.2. Brains were collected, processed and sectioned as previously described. ${ }^{42}$

Immunofluorescence \& in situ hybridization. Ten $\mu \mathrm{m}$ thick sections were washed for $5 \mathrm{~min}$, three times in PBS and then incubated in the blocking mix (PBS $1 \times /$ FBS $10 \% / B S A 1 \mathrm{mg} / \mathrm{ml} /$ Triton $100 \times 0.1 \%$ ) for $1 \mathrm{~h}$ at room temperature. Antibodies were diluted in blocking mix and incubated at $+4{ }^{\circ} \mathrm{C}$ overnight, as suggested by the manufacturer's instructions. The following day, sections were washed in PBS for $5 \mathrm{~min}$, three times, and fluorescent secondary antibodies diluted in blocking mix (concentration suggested by the manufacturer's instructions) were applied. Slides were washed three times in PBS for $5 \mathrm{~min}$ each and then incubated in 4'-6-diamidino-2-phenylindole (Dapi, Roche, Milan Italy) solution for nuclei counterstaining. When necessary, antigens were unmasked by boiling samples in 10-mM sodium citrate $(\mathrm{pH} \mathrm{6}$ ) for $5 \mathrm{~min}$ Immunohistochemistry was performed as previously described. ${ }^{43}$ Briefly, slices were incubated in $\mathrm{H}_{2} \mathrm{O}_{2} 3 \%$ for 20 min before adding the blocking mix. Antibodies were diluted in blocking mix and incubated at $+4^{\circ} \mathrm{C}$ overnight, as suggested by the manufacturer's instructions. The following day, sections were washed in PBS three times, and the biotin-conjugated secondary antibody (Vector labs, Milan, Italy) was applied for $2 \mathrm{~h}$. Then sections were washed before adding the avidinHRP reagent (Vector). Signals were revealed by incubating slices with 3-amino-9ethylcarbazole (AEC, Sigma) solution.

The following antibodies were used: rabbit $\alpha$-GFAP (1:1500, Dako, Milan, Italy), rabbit $\alpha$-pH3 (1:200, Millipore, Milan, Italy), chicken $\alpha$-GFP (1:1000, Abcam, Cambridge, UK), rabbit $\alpha$-GFP $(1: 1000$, Molecular Probes, life technologies, Milan, Italy), rabbit $\alpha-p S m a d 1 / 5 / 8$ (1:500, Cell Signalling, Danvers, MA, USA), rabbit $\alpha$-CR $(1: 300$, Swant, Marly, Switzerland), rat $\alpha$-BrdU (1:500, Abcam), mouse $\alpha$-BrdU (1:100, BD, Milan, Italy), click-it EdU AlexaFluor 595 and 488 Imaging reagents (Invitrogen), rabbit $\alpha$-Tbr2 $(1: 500$, Abcam), mouse $\alpha$-TuJ1 (1:1500, Millipore), rabbit $\alpha$-Ki67 (1:100, Novocastra, Newcastle, UK), goat $\alpha$-Dcx (1:100, Santa Cruz Biotechnology, Inc., Heidelberg, Germany), mouse $\alpha$-RC2 (1:100, Hybridoma bank System, lowa City, IA, USA), goat $\alpha$-Prox1 (1:400, R\&D System, Milan, Italy), rabbit $\alpha$-Casp3a $(1: 100$, NEB, Hitchin, UK), rabbit $\alpha$-RFP $(1: 500$, MBL, Cornaredo, Italy), rabbit $\alpha$-Blbp (1:700, Millipore), mouse $\alpha$-NeuN (1:1000, Chemicon, Milan, Italy), rabbit $\alpha$-Olig2 (1:500, Immunological Science, Rome, Italy). Appropriate fluorophore-conjugated secondary antibodies (Alexa-Fluor 488, 546 and 633 Molecular Probes) or biotinylated secondary antibodies were used. Nuclei were stained with Dapi (Roche). Light (Olympus, Milan, Italy, BX51 with $4 \times$ and $20 \times$ objectives) and confocal (Leica, Wetzlar, Germany, SP5 with $40 \times$ objectivemicroscopy was performed to analyze tissues and cell staining. Analyses were performed by using Leica LCS lite software and Adobe Photoshop CS software.

Briefly, ten $\mu$ m-tick brain sections were postfixed $15 \mathrm{~min}$ in $4 \%$ paraformaldehyde then washed three times in PBS. Slides were incubated in $0.5 \mathrm{mg} / \mathrm{ml}$ of Proteinase $\mathrm{K}$ in $100 \mathrm{mM}$ Tris- $\mathrm{HCl}(\mathrm{pH} 8), 50-\mathrm{mM}$ EDTA for $10 \mathrm{~min}$ at $30^{\circ} \mathrm{C}$. This was followed by $15 \mathrm{~min}$ in $4 \%$ paraformaldehyde. Slices were then washed three times in PBS, then washed in $\mathrm{H}_{2} \mathrm{O}$. Sections were incubated in Triethanolamine $0.1 \mathrm{M} \mathrm{(pH} \mathrm{8)} \mathrm{for} 5 \mathrm{~min}$, then $400 \mu \mathrm{l}$ of acetic anhydride was added two times for $5 \mathrm{~min}$ each. Finally, sections were rinsed in $\mathrm{H}_{2} \mathrm{O}$ for $2 \mathrm{~min}$ and air-dried. Hybridization was performed overnight at $60^{\circ} \mathrm{C}$ with $\mathrm{P}^{33}$ riboprobes at a concentration ranging from $10^{6}$ to $10^{7}$ counts per minute $(\mathrm{cpm})$. The following day, sections were rinsed in SSC $5 \times$ for $5 \mathrm{~min}$, then washed in Formamide $50 \%$ SSC $2 \times$ for $30 \mathrm{~min}$ at $60^{\circ} \mathrm{C}$. Then slides were incubated in Ribonuclease-A (Roche) $20 \mu \mathrm{g} / \mathrm{ml}$ in $0.5 \mathrm{M} \mathrm{NaCl}, 10 \mathrm{mM}$ Tris-HCl (pH 8), $5 \mathrm{mM}$ EDTA $30 \mathrm{~min}$ at $37^{\circ} \mathrm{C}$. Sections were washed in Formamide $50 \%$ SSC $2 \times$ for 30 min at $60^{\circ} \mathrm{C}$, then slides were rinsed two times in SSC $2 \times$. Finally, slides were dried by using Ethanol series. Lm1 (GE Healthcare Life Sciences, Milan,
Italy) emulsion was applied in dark room, according to the manufacturer's instructions. After 1 week, sections were developed in dark room, counterstained with Dapi and mounted with DPX (BDH) mounting solution. The following probes were used: mouse HtrA1 probe was generated by cloning the entire $3^{\prime} U T R$ region of the gene according to the information available in GenBank (accession number NM_019564). Sense probes, showing no signals, were used as negative controls. Mouse Msx1 probe was generated by cloning the $3^{\prime}$ UTR region of the gene according to the information available in GenBank (accession number NM_010835). Nonradioactive in situ hybridizations were performed as described previously. ${ }^{41,44}$ $\alpha$-Crystallin in situ probe was PCR-amplified; GenBank accession number AF039391; nucleotides 392-1192 (a gift from N. Funatsu, Tokyo, Japan), FoxG1 riboprobe was generated according to the data available in GenBank (NM_001160112). ${ }^{44}$

Tunel assay. Ten $\mu \mathrm{m}$ thick sections from E14.5, E16.5, E18.5 and P15 forebrains were postfixed in $4 \%$ paraformaldehyde, then incubated in Proteinase $\mathrm{K}$ buffer for $5^{\prime}$ at room temperature and then exposed to $10 \mathrm{mg} / \mathrm{ml}$ Proteinase $\mathrm{K}$ for $15^{\circ} \mathrm{C}$ at room temperature. Reaction was stopped by washing samples three times in PBS $1 \times$. Slices were then incubated with Terminal Transferase buffer for $15^{\circ} \mathrm{C}$ before adding the following reagents: $10-\mu \mathrm{g} / \mathrm{ml}$ Biotin 16 -dUTP, $1-\mathrm{mm} \mathrm{CoCl} 2$ and $10 \mathrm{U} / \mathrm{ml}$ of Terminal Transferase (Roche). Reaction was incubated $1 \mathrm{~h}$ at $37^{\circ} \mathrm{C}$ and stopped with $\mathrm{H}_{2} \mathrm{O}$. Endogenous peroxidase quenching was achieved by incubating slices in $0.1 \% \mathrm{H}_{2} \mathrm{O}_{2}$ for $15^{\circ} \mathrm{C}$. Slices were incubated in blocking buffer $(10 \%$ FBS, PBS $1 \times)$ for $15^{\circ} \mathrm{C}$ and then incubated in Streptoavidin/Biotin amplification kit (Vector) for $2 \mathrm{~h}$. Reaction product was visualized with $0.05 \%$ AEC and $0.005 \% \mathrm{H}_{2} \mathrm{O}_{2}$. Positive controls were obtained by incubating slices in $3 \mathrm{U} / \mathrm{ml} \mathrm{DNase}$ for $15^{\circ} \mathrm{C}$ at room temperature.

Microarrays analysis. PO forebrains were micro-dissected to obtain cortical and hippocampal mRNA preparations. The Figures $3 a$ and $b$ depicts the adopted statistical and bioinformatic workflow. Total RNAs from the cortex and hippocampus of WT and Gfap ${ }^{C r e}$ Dicer/f mice were extracted by using RNeasy Mini Kit (Qiagen) according to the manufacturer's instructions. cRNAs probes were generated by using Illumina total prep RNA amplification kit (Ambion/Life Technologies, Milan, Italy) according to the manufacturer's instructions. Hybridization on Illumina MouseWG-6_V2 arrays (Illumina, Eindhoven, Netherlands) was performed according to the manufacturer's protocol and image files were acquired with Illumina Bead-express scanner. After data normalization and filtering, the LIMMA algorithm identified the genes differentially expressed in Gfap ${ }^{\text {Cre }}$ Dicert/f versus WT brains passing the significance threshold of $<0.01$ and displaying a minimum fold change of \pm 1.7 . Unsupervised hierarchical clustering of the samples showed that the transcriptional signatures classified correctly the samples derived from WT and Gfap ${ }^{C r e}$ Dicer ${ }^{t / f}$ mice, both in the cortex and in the hippocampus (Figure 3). The array contained 45281 probes representing transcripts contained in the NCBI-Refseq, Riken, and Meebo databases. The raw data were background subtracted and cubic spine normalized using Illumina GenomeStudio-GX software. Probes were filtered according to the following criteria: detection $P$-value $<0.05$ in at least $50 \%$ of the samples. No outlier samples were found in PCA (Principal Component Analysis) and hierarchical sample clustering. Differential gene expression analyses were performed independently for the cortex and hippocampus data using one Channel GUI package implemented in the R-Bioconductor platform. ${ }^{45}$ The LIMMA algorithm was used to compute a linear model fit. ${ }^{46}$ Gene prioritization in the signature related to each tissue was then obtained using the biomarker filter module of Ingenuity software (criteria: organism = mouse, tissue of expression = nervous system and upregulated genes with 2.5 or more fold change). The miRNA enrichment analysis was performed in Genecodis tool. ${ }^{47}$

In vitro and in vivo Luciferase assay. Hek293T cells $\left(5 \times 10^{4} /\right.$ well) were seeded in 24-well plates and transfected (DharmaFect Duo transfecting reagent, Dharmacon, Thermo Scientific, Lafayette, CO, USA) with pCAG-RlucHtrA1-3'UTR (0.05 $\mu \mathrm{g} / \mathrm{well})$, pcDNA3.1-luc-firefly $(0.025 \mu \mathrm{g} /$ well), and increasing concentration of miRIDIAN microRNA Mimics (Thermo Scientific, Lafayette, CO, USA) encoding: cel-miR-67 (negative control miRNA), miR-30e, miR-149 and miR181d. Mimics were used at the following concentration: $0.1,5$ and $20 \mathrm{pmol} /$ well. pCAG-Rluc-HtrA1-3'UTR plasmids containing point mutations in the target sites for either miR-30e (5'-AGTTtttgCAAATGT3') and miR-181d (5'-ugGTGGCTGcagcTTACTTACAa- $\left.3^{\prime}\right)$ were included in each experiments as negative controls. (Bold letters indicate mutated sequences in the $3^{\prime}$ UTR of 
pCAG-Rluc-HtrA1-30UTR vector that inhibit binding of miR-30 e and miR-181d). Transfection efficiency was estimated by performing parallel transfection with Dy547labeled miRIDIAN microRNA Mimic Transfection Control, at the concentration of $5 \mathrm{pmol} / \mathrm{well}$. Measurement of luciferase activity was done by using the dual-luciferase system (Promega, Milan, Italy) on a luminometer (GloMax 20/20 Luminometer; Promega). Relative Renilla luciferase activity was reported as a ratio of Renilla over Firefly luciferase readouts. In vivo luciferase assays were performed after in utero electroporation of the dorsal telencephalon of E13.5 wild-type (WT) CD1 mouse embryos (Charles River laboratories, Italy) with pCAG-driven reporter plasmids (pCAGRluc-HtrA1-3'UTR or pCAG-Rluc-empty, and pCAG-Fluc and pCAG-mCherry, each at final concentration of $1 \mu \mathrm{g} / \mu \mathrm{l})$. Embryos were harvested after $8 \mathrm{~h}$ of in utero development and processed for luciferase assays as described.

Real-time and standard RT-PCR. Total RNA was extracted by using RNeasy Mini Kit (Qiagen) according to the manufacturer's recommendations including DNase (Promega) digestion. cDNA synthesis was performed by using ThermoScript RT-PCR System (Invitrogen) and Random Hexamer (Invitrogen), according to the manufacturer's instructions in final volume of $20 \mu \mathrm{l}$. The LightCycler 480 System (Roche) and SYBR Green JumpStart Taq ReadyMix for High Throughput Q-PCR (Sigma) were used for real-time PCR. CDNA analysis was also done by standard RT-PCR, performed on Bio-Rad C-1000 thermal cycler (38 cycles, $95^{\circ} \mathrm{C}-1^{\prime}, 65^{\circ} \mathrm{C}-30^{\prime}, 72^{\circ} \mathrm{C}-30^{\prime}$ ). Each sample was normalized by using the housekeeping gene Histone $\mathrm{H} 3$ with the following primers: $\mathrm{H} 3 \mathrm{~F}$ : 5'-GGTGAAGAAACCTCATCGTTACAGGCCTGGTAC-3' H3 R: 5'-CTGCAAAGC ACCAATAGCTGCACTCTGGAAGC-3'. Specific primers were used for gene expression analysis: HtrA1 F: 5'-GGCCTCGGCCACAGTACGACG-3'; HtrA1 R: 5'-GCGATCTTCTCCACCACATCAGC-3'; Dicer F: 5'-TTTGGACTACCTCATAAC CAAGCACC-3', Dicer R: $5^{\prime}$-CAGAGTCCATTCCTTGCATTTCGTTC-3'. FoxG1 F: 5'-CACCCCATGCCCTACAGCTCCG-3'; FoxG1 R: 5'-AGCAGGTTGACGGAGC AGGGG-3'. Total miRNA and RNA extracts were obtained from tissues by using miRNeasy Mini kit (Qiagen, Milan, Italy) according to the manufacturer's recommendations. RT was done by using miScript reverse transcription kit (Qiagen) and amplifications were done by using miScript primer assay and miScript Syber green (Qiagen) kits according to the manufacturer's recommendations. The following miScript primers were used: Mm_miR-30e, Mm_miR-149, Mm_miR-181d, Normalization of each real-time PCR was done by using Hs_RNU1a and Hs_RNU6b primers.

In-utero electroporation. IUE was performed as previously described. ${ }^{48}$ Briefly, the following plasmids were introduced into progenitor cells of E13.5 and E14.5 forebrains (CD1, Charles River): pCAG-GFP $(0.1 \mu \mathrm{g} / \mu \mathrm{l})$, pcDNA5-HtrA1 $(1 \mu \mathrm{g} / \mu \mathrm{l})$. Plasmids were mixed with $0.01 \%$ Fast green (Sigma) and $1-2 \mu \mathrm{l}$ of each DNA mix were injected into the ventricle through a fine-glass capillary. Electrodes (Tweezertrodes, BTX Harvard Apparatus, Holliston, MA, USA) were placed flanking the ventricular region of each embryo, covered by a drop of PBS and pulsed 4 times at $40 \mathrm{~V}$ for $50 \mathrm{~ms}$, separated by intervals of $950 \mathrm{~ms}$, with a square wave electroporator (ECM 830, BTX Harvard Apparatus). Then, the uterine horn was placed back into the abdominal cavity filled with warm PBS $1 \times$. Brains electroporated at E13.5 were collected at E15.5, whereas embryos electroporated at E14.5 were collected at E17.5. RG cell morphology was evaluated by electroporating the PCAG-Cherry plasmid $(0.1 \mu \mathrm{g} / \mu \mathrm{l})$ in control and Gfap ${ }^{\text {Cre }}$ Dicer $^{\mathrm{f} / \mathrm{f}}$ embryos at E16.5. Embryos were collected at E18.5 and processed for the visualization of the Cherry protein by immunofluorescence. Tissues were fixed in $4 \%$ paraformaldehyde as described above. Only embryos showing comparable electroporated patches were included in the analysis. E14.5 Gfap ${ }^{\text {Cre }}$ Dicer fft $^{\mathrm{fth}}$ embryos were electroporated with pCAG-Cherry $(0.1 \mu \mathrm{g} / \mu \mathrm{l})$ and mimics encoding miR-30e, -181d or cel-miR-67 (negative control miRNA), at the concentration of 5 pmol. Embryos were collected after 3 days and processed as described above. Electroporation efficiency was evaluated by electroporating Dy547-labeled miRIDIAN microRNA Mimic Transfection Control.

Statistics. Bar graphs represent mean values \pm S.E.M. or mean values \pm S.D. Data were analyzed as appropriate by Student's $t$ and Anova tests by using Graph-Pad Prism version 4. A Significance was accepted when $P<0.05$.

\section{Conflict of Interest}

The authors declare no conflict of interest.
Acknowledgements. We acknowledge Dr. R. Furlan, B. Borgiani and Dr. P. Brown for helpful discussion and suggestions. Annamaria Nigro conducted this study on partial fulfilment of her PhD in Molecular Medicine, Program in Experimental Neurology at San Raffaele University, Milan, Italy. We thank Dr. B. Harfe for Dicer transgenic mice, Dr. V. Shridhar for HtrA1-expressing plasmids.

\section{Author contributions}

LM and GM designed the study; AN, RM, AB performed experiments; DPT and YMC performed luciferase experiments; RM and $\mathrm{CF}$ carried out computational analyses; $M E$ and $A B$ contributed to in vivo characterization of HtrA1; LM, DPT, AN and CF analyzed the data; GM and GC provided the financial support for the study; LM wrote the paper, and all listed authors commented and corrected on article drafts. This work was supported by PRIN, grant number R0467.

1. Gotz M, Huttner WB. The cell biology of neurogenesis. Nat Rev Mol Cell Biol 2005; 6: 777-788.

2. Grove EA, Tole S, Limon J, Yip L, Ragsdale CW. The hem of the embryonic cerebral cortex is defined by the expression of multiple Wnt genes and is compromised in Gli3-deficient mice. Development 1998; 125: 2315-2325.

3. Muzio L, Mallamaci A. Emx1, emx2 and pax6 in specification, regionalization and arealization of the cerebral cortex. Cereb Cortex 2003; 13: 641-647.

4. Saba R, Schratt GM. MicroRNAs in neuronal development, function and dysfunction. Brain Res 1338: 3-13.

5. Krol J, Busskamp V, Markiewicz I, Stadler MB, Ribi S, Richter J et al. Characterizing light-regulated retinal microRNAs reveals rapid turnover as a common property of neuronal microRNAs. Cell 141: 618-631.

6. Candiani S, Moronti L, De Pietri Tonelli D, Garbarino G, Pestarino M. A study of neural-related microRNAs in the developing amphioxus. Evodevo 2: 15.

7. Friedman RC, Farh KK, Burge CB, Bartel DP. Most mammalian mRNAs are conserved targets of microRNAs. Genome res 2009; 19: 92-105.

8. Guo H, Ingolia NT, Weissman JS, Bartel DP. Mammalian microRNAs predominantly act to decrease target mRNA levels. Nature 466: 835-840.

9. Kim VN. MicroRNA precursors in motion: exportin-5 mediates their nuclear export. Trends Cell Biol 2004; 14: 156-159.

10. Hammond SM. Dicing and slicing: the core machinery of the RNA interference pathway. FEBS letters 2005; 579: 5822-5829.

11. Zhuo L, Theis M, Alvarez-Maya I, Brenner M, Willecke K, Messing A. hGFAP-cre transgenic mice for manipulation of glial and neuronal function in vivo. Genesis $2001 ; 31$ : 85-94.

12. Soriano P. Generalized lacZ expression with the ROSA26 Cre reporter strain. Nat genet 1999; 21: 70-71.

13. Harfe BD, McManus MT, Mansfield JH, Hornstein E, Tabin CJ. The RNaselll enzyme Dicer is required for morphogenesis but not patterning of the vertebrate limb. Proc Natl Acad Sci USA 2005; 102: 10898-10903.

14. De Pietri Tonelli D, Pulvers JN, Haffner C, Murchison EP, Hannon GJ, Huttner WB. miRNAs are essential for survival and differentiation of newborn neurons but not for expansion of neural progenitors during early neurogenesis in the mouse embryonic neocortex. Development 2008; 135: 3911-3921.

15. Oldekamp J, Kraemer N, Alvarez-Bolado G, Skutella T. bHLH gene expression in the Emx2-deficient dentate gyrus reveals defective granule cells and absence of migrating precursors. Cereb Cortex 2004; 14: 1045-1058.

16. Funatsu N, Inoue T, Nakamura S. Gene expression analysis of the late embryonic mouse cerebral cortex using DNA microarray: identification of several region- and layer-specific genes. Cereb Cortex 2004; 14: 1031-1044.

17. Nowakowski TJ, Mysiak KS, Pratt T, Price DJ. Functional dicer is necessary for appropriate specification of radial glia during early development of mouse telencephalon. PLOS ONE 6: e23013.

18. Kawase-Koga Y, Otaegi G, Sun T. Different timings of Dicer deletion affect neurogenesis and gliogenesis in the developing mouse central nervous system. Dev Dyn 2009; 238: 2800-2812.

19. Davis TH, Cuellar TL, Koch SM, Barker AJ, Harfe BD, McManus MT et al. Conditional loss of Dicer disrupts cellular and tissue morphogenesis in the cortex and hippocampus. $J$ Neurosci 2008; 28: 4322-4330.

20. Hartfuss E, Galli R, Heins N, Gotz M. Characterization of CNS precursor subtypes and radial glia. Dev biol 2001; 229: 15-30.

21. Takahashi T, Nowakowski RS, Caviness VS Jr. Cell cycle parameters and patterns of nuclear movement in the neocortical proliferative zone of the fetal mouse. J Neurosci 1993; 13: $820-833$.

22. Englund C, Fink A, Lau C, Pham D, Daza RA, Bulfone A et al. Pax6, Tbr2, and Tbr1 are expressed sequentially by radial glia, intermediate progenitor cells, and postmitotic neurons in developing neocortex. J Neurosci 2005; 25: 247-251.

23. Edwards MA, Yamamoto M, Caviness VS Jr. Organization of radial glia and related cells in the developing murine CNS. an analysis based upon a new monoclonal antibody marker. Neuroscience 1990; 36: 121-144.

24. Young KM, Fogarty M, Kessaris N, Richardson WD. Subventricular zone stem cells are heterogeneous with respect to their embryonic origins and neurogenic fates in the adult olfactory bulb. J Neurosci 2007; 27: 8286-8296. 
25. Nait-Oumesmar B, Picard-Riera N, Kerninon C, Baron-Van Evercooren A. The role of SVZderived neural precursors in demyelinating diseases: from animal models to multiple sclerosis. J neurol sci 2008; 265: 26-31.

26. Andersson T, Rahman S, Sansom SN, Alsio JM, Kaneda M, Smith J et al. Reversible block of mouse neural stem cell differentiation in the absence of dicer and microRNAs. PLOS ONE 5: e13453.

27. Oka C, Tsujimoto R, Kajikawa M, Koshiba-Takeuchi K, Ina J, Yano M et al. HtrA1 serine protease inhibits signaling mediated by Tgfbeta family proteins. Dev 2004; 131 1041-1053.

28. Anderson RM, Lawrence AR, Stottmann RW, Bachiller D, Klingensmith J. Chordin and noggin promote organizing centers of forebrain development in the mouse. Development 2002; 129: 4975-4987.

29. Caronia G, Wilcoxon J, Feldman P, Grove EA. Bone morphogenetic protein signaling in the developing telencephalon controls formation of the hippocampal dentate gyrus and modifies fear-related behavior. J Neurosci 30: 6291-6301.

30. Hebert JM, Mishina Y, McConnell SK. BMP signaling is required locally to pattern the dorsal telencephalic midline. Neuron 2002; 35: 1029-1041.

31. Panchision DM, Pickel JM, Studer L, Lee SH, Turner PA, Hazel TG et al. Sequential actions of BMP receptors control neural precursor cell production and fate. Genes Dev 2001; 15: 2094-2110.

32. Chen D, Zhao M, Mundy GR. Bone morphogenetic proteins. Growth Factors 2004; 22 233-241.

33. Furuta Y. Piston DW, Hogan BL. Bone morphogenetic proteins (BMPs) as regulators of dorsal forebrain development. Development 1997; 124: 2203-2212.

34. von Bubnoff A, Peiffer DA, Blitz IL, Hayata T, Ogata S, Zeng $Q$ et al. Phylogenetic footprinting and genome scanning identify vertebrate BMP response elements and new target genes. Dev Biol 2005; 281: 210-226.

35. He X, Ota T, Liu P, Su C, Chien J, Shridhar V. Downregulation of HtrA1 promotes resistance to anoikis and peritoneal dissemination of ovarian cancer cells. Cancer Res 70 : 3109-3118.

36. De Luca A, De Falco M, De Luca L, Penta R, Shridhar V, Baldi F et al. Pattern of expression of HtrA1 during mouse development. J Histochem Cytochem 2004; 52: 1609-1617.

37. Li Q, Bian S, Hong J, Kawase-Koga Y, Zhu E, Zheng Y et al. Timing specific requirement of microRNA function is essential for embryonic and postnatal hippocampal development. PLOS ONE 6: e26000.

38. Clausen T, Southan $\mathrm{C}$, Ehrmann M. The HtrA family of proteases: implications for protein composition and cell fate. Mol cell 2002; 10: 443-455.
39. Baldi A, De Luca A, Morini M, Battista T, Felsani A, Baldi F et al. The HtrA1 serine protease is down-regulated during human melanoma progression and represses growth of metastatic melanoma cells. Oncogene 2002; 21: 6684-6688.

40. Chien J, Ota T, Aletti G, Shridhar R, Boccellino M, Quagliuolo $L$ et al. Serine protease HtrA1 associates with microtubules and inhibits cell migration. Mol Cell Biol 2009; 29: 4177-4187.

41. Muzio L, DiBenedetto B, Stoykova A, Boncinelli E, Gruss P, Mallamaci A. Conversion of cerebral cortex into basal ganglia in Emx2 $(-/-)$ Pax6(Sey/Sey) double-mutant mice. Nat Neurosci 2002; 5: 737-745.

42. Marinaro C, Butti E, Bergamaschi A, Papale A, Furlan R, Comi G et al. In vivo fate analysis reveals the multipotent and self-renewal features of embryonic AspM expressing cells. PLOS ONE 6: e19419.

43. Muzio L, Cavasinni F, Marinaro C, Bergamaschi A, Bergami A, Porcheri C et al. Cxcl10 enhances blood cells migration in the sub-ventricular zone of mice affected by experimental autoimmune encephalomyelitis. Mol Cell Neurosci 2010; 43: 268-280.

44. Muzio L, Mallamaci A. Foxg1 confines Cajal-Retzius neuronogenesis and hippocampal morphogenesis to the dorsomedial pallium. J Neurosci 2005; 25: 4435-4441.

45. Sanges R, Cordero F, Calogero RA. oneChannelGUI: a graphical interface to Bioconductor tools, designed for life scientists who are not familiar with R language. Bioinformatics 2007; 23: 3406-3408.

46. Smyth GK. Linear models and empirical bayes methods for assessing differential expression in microarray experiments. Stat Appl Genet Mol Biol 2004; 3: 1544-6115.

47. Nogales-Cadenas R, Carmona-Saez $\mathrm{P}$, Vazquez M, Vicente $\mathrm{C}$, Yang $\mathrm{X}$, Tirado $\mathrm{F}$ et al. GeneCodis: interpreting gene lists through enrichment analysis and integration of diverse biological information. Nucleic Acids Res 2009; 37(Web Server issue): W317-W322.

48. Marinaro C, Pannese M, Weinandy F, Sessa A, Bergamaschi A, Taketo MM et al. Wnt Signaling Has Opposing Roles in the Developing and the Adult Brain That Are Modulated by Hipk1. Cereb Cortex 17.

Cell Death and Disease is an open-access journal published by Nature Publishing Group. This work is licensed under the Creative Commons Attribution-NonCommercialShare Alike 3.0 Unported License. To view a copy of this license, visit http://creativecommons.org/licenses/by-nc-sa/3.0/

Supplementary Information accompanies the paper on Cell Death and Disease website (http://www.nature.com/cddis) 